\title{
GUIDELINES FOR A \\ Space Propulsion Device \\ Based on HeIM's Quantum Theory
}

Walter Dröscher', Jochem Häuser ${ }^{1,2}$

${ }^{1}$ Institut für Grenzgebiete der Wissenschaft (IGW),

Leopold - Franzens Universität Innsbruck, Innsbruck, Austria

${ }^{2}$ Department of Transportation, University of Applied Sciences and

Department of High Performance Computing, CLE GmbH, Salzgitter, Germany

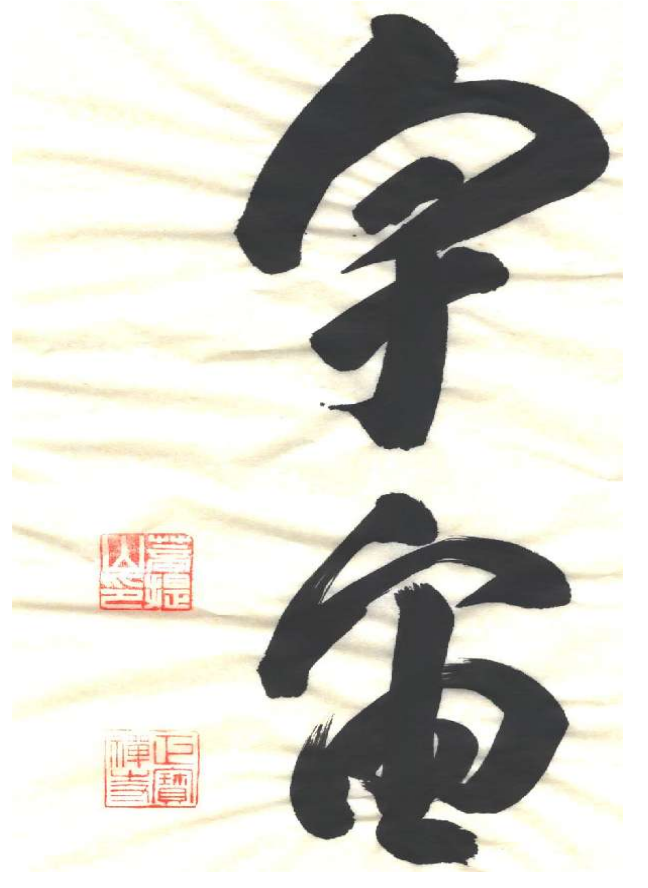

40 $^{\text {тн }}$ AIAA/ASME/SAE/ASEE

Joint Propulsion Conference \& Exhibit, Fort Lauderdale, Florida, 11-14 JuLY, 2004 
The text of the calligraphy on the front page means Cosmos, comprising the two chinese symbols for space and time. This calligraphy was done by Hozumi Gensho Roshi, Professor of Applied Sciences at Hanazono University, Kyoto, Japan in September 2003. The two red squares depict the seal of Hozumi Gensho Roshi.

2004

Institut für Grenzgebiete der Wissenschaft, Leopold - Franzens Universität Innsbruck, Innsbruck, Austria 


\section{Table of Contents}

1 Space Propulsion and Higher-Dimension Quantized Spacetime Physics...............................4



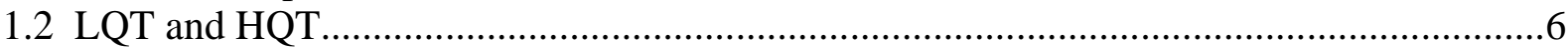

1.3 Fundamental Physical Interactions in 8-D Quantized Space.....................................

2 The Physical Principles for Field Propulsion ...................................................................... 7

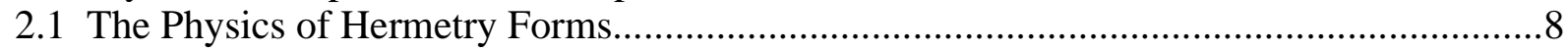

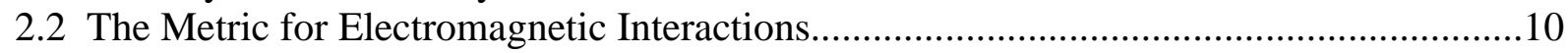

2.3 The Metric for Coupling Electromagnetism and Gravitation.......................................11

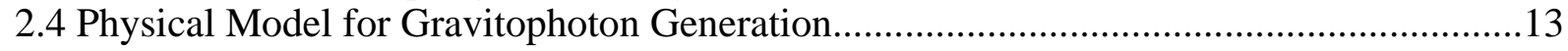

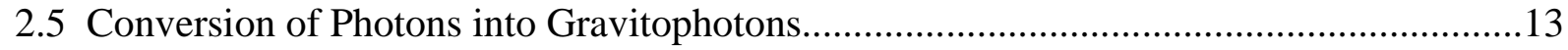

3 Space Flight Dynamics of Gravitophoton Field Propulsion.................................................14

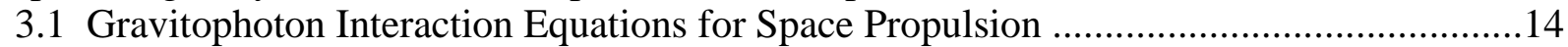

3.2 Technical Data for Acceleration Gravitophoton Field Propulsion ….............................16

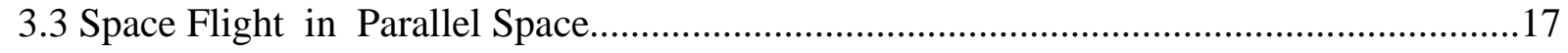

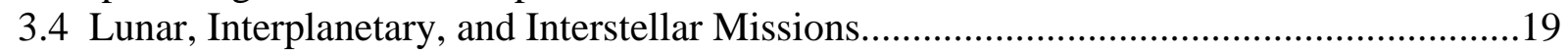

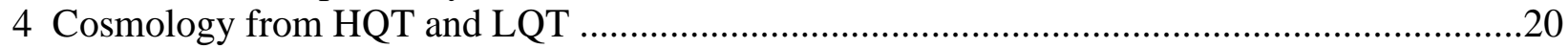

4.1 Deficiencies in Current Fundamental Physical Theories............................................20

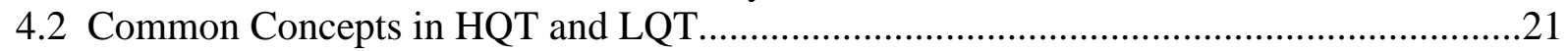

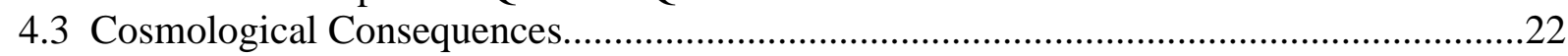

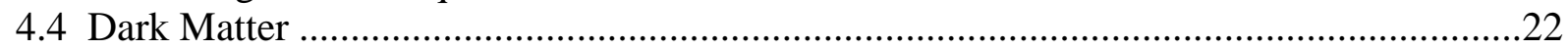

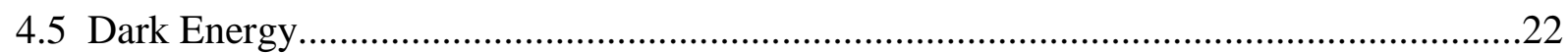



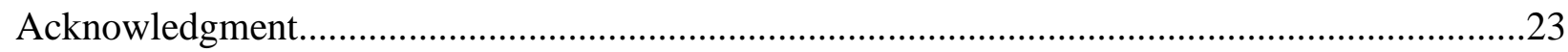

Appendix A: Mass Spectrum of Elementary Particles........................................................24

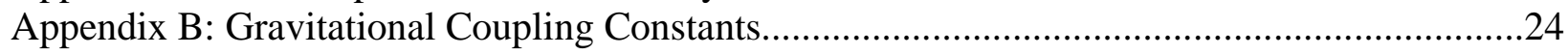

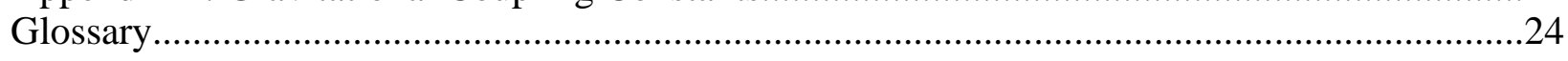

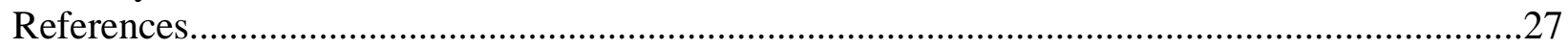




\begin{abstract}
This paper is the third one in a series of publications, describing a novel and revolutionary space propulsion technique, based on a unified field theory in a quantized, higher-dimensional space, developed by the late B. Heim and the first author, termed Heim quantum theory $(H Q T)$ in the following. It is interesting to note that this theory shares a similar physical picture, namely a quantized spacetime, with the recently published loop quantum theory $(L Q T)$ by L. Smolin, A. Ashtektar, C. Rovelli, M. Bojowald et al. [11, 24-28]. LQT, if proved correct, would stand for a major revision of current physics, while $H Q T$ would cause a revolution in the technology of propulsion.
\end{abstract}

For effective and efficient interplanetary as well as interstellar travel, NASA's Breakthrough Propulsion Physics Program (BPP) specified three basic features, namely little or no fuel mass, limited amount of energy consumption (a spacecraft approaching the speed of light would not satisfy this requirement, since its mass becomes infinite), and (preferably) superluminal speed. To satisfy these requirements a revolution in space propulsion technology is needed. Such breakthrough propulsion techniques can only emerge from novel physics. If we believed that current physics held the answer to all questions, a BPP device would not be possible. Recently, however, more and more evidence has been piling up that current physics is far from final answers and, in addition, exhibits fundamental inconsistencies, even on the classical level. Furthermore, quantum theory $(Q T)$ in its current form does not lead to an explanation of the elementary structures of matter, and does not lead to a consistent cosmology either.

For a revolutionary space transportation system, however, the physical concepts of matter and inertia as well as the nature of space and time have to be understood. In $Q T$ the existence of matter is taken for granted, defining an elementary particle as a point-like structure [17]. In classical physics, including the General Theory of Relativity $(G R)$, science starts from the belief that space and time are infinitely divisible, in other words, that spacetime is continuous (a differentiable manifold in the mathematical sense). Both ideas contradict Nature's all pervading quantization principle and immediately lead to contradictions in the form of infinite self-energies etc. or self-accelerations [18]. HQT is an extension of Einstein's $G R$, using his field equations as a template in a quantized higher-dimensional space, but also extending these equations into the subatomic range. This eventually leads to a poly-metric, whose partial metric structures are interpreted as fundamental physical interactions. This theory, seems to complement both $Q T$ and $G R$, in explaining the nature of elementary particles as well as their discrete mass spectrum and life times, based on the basis of a quantized geometrodynamics (quantized elemental surfaces of some $10^{-70} \mathrm{~m}^{2}$, termed metron by Heim) in a 12 dimensional space. Heim derives a dimensional law that determines the maximum number of possible dimensions that can exist, along with admissible subspaces, and also gives their physical interpretation. $H Q T$ seems to be able to explain the nature of matter (physicists deemed this question to be of importance in the early fifties, see [21]). The physical features of the postulated 12 dimensional, quantized hyperspace, denoted as Heim space by the authors, is described in detail. The 12D Heim space comprises five semantic units, namely, the subspaces $\mathbb{R}^{3}$ (space), $\mathrm{T}^{1}$ (time), $\mathrm{S}^{2}$ (organization), $\mathrm{I}^{2}$ (information), and $\mathrm{G}^{4}$ (steering of $\mathrm{I}^{2}$ ) where superscripts denote dimension. Except for the 3 spatial dimensions, all other coordinates are imaginary. Several metric tensors can be constructed from these subspaces. Each metric tensor is associated with a specific physical interaction, similar to Einstein's GR, where spacetime curvature is interpreted as gravitation (graviton). Analyzing the metric tensors acting in $\mathbb{R}^{4}$, the theory predicts six fundamental interactions, instead of the four experimentally known ones. These interactions represent physical fields that are carrying energy. According to $H Q T$, a transformation of electromagnetic energy into gravitational energy should be possible. It is this interaction that is used as the physical basis for the novel space propulsion concept, termed field propulsion [1, 2], which is not conceivable within the framework of current physics.

The paper comprises four technical chapters. In the first chapter, a qualitative discussion of the six fundamental interactions, derived from the concept of Heim space and its conse- 
quences for a novel propulsion system, are presented. In addition, a qualitative discussion of the physical principle that serves as the basis for advanced propulsion is given. In chapter two, the physical principles of the so called field propulsion system are quantitatively addressed, explaining their application in future spaceflight ${ }^{2}$. In particular, it is shown how the poly-metric from Heim space leads to a metric describing electromagnetic phenomena and its conversion into a gravitational ${ }^{3}$ like metric, postulating a novel particle, the gravitophoton. In chapter three, the equations of the gravitophoton interaction are derived, and a physical model is presented to calculating the magnitude of the gravitophoton interaction. This is also the main chapter, presenting the quantitative physical model of the gravitophoton interaction and the concept of parallel space from which the physical guidelines for the field propulsion device can be derived. In particular, the technical requirements for a gravitophoton propulsion device will be discussed. The experimental set up of such a device will also be presented. In addition, a lunar mission, an interplanetary, and an interstellar mission will be investigated. In chapter four, similarities between HQT and LQT are discussed. Cosmological consequences dealt with concern the amount of dark matter (concept of parallel space) and the cause of dark energy. The acceleration of the cosmic expansion is explained qualitatively, since it turns out that the postulated sixth fundamental force (interpreted as quintessence) and represented by the postulated vacuum particle, is of repulsive nature.

\section{Nomenclature and physical constants}

$\tilde{A}$ value for the onset of conversion of photons into gravitophotons, see Eq. (39).

A denotes the strength of the shielding potential caused by virtual electrons, see Eq. (37).

\section{Compton wave length of the electron}

$$
\lambda_{C}=\frac{h}{m_{e} c}=2.43 \times 10^{-12} m, \quad \lambda_{C}=\lambda_{C} / 2 \pi .
$$

2 This chapter contains a certain amount of mathematics. The reader may wish to skip the derivations and continue with the gravitational Heim-Lorentz force, Eq. (47).

3 The term gravitational is reserved to the two additional interactions represented by the gravitophoton and the vacuum particle acting on material particles. c speed of light in vacuum $299,742,458 \mathrm{~m} / \mathrm{s}$, $\left(1 / c^{2}=\varepsilon_{0} \mu_{0}\right)$.

$D$ diameter of the primeval universe, some $10^{125} \mathrm{~m}$, that contains our optical universe.

$D_{\mathrm{O}}$ diameter of our optical universe, some $10^{26} \mathrm{~m}$.

$d$ diameter of the rotating torus, see caption Table .

$d_{\mathrm{T}}$ vertical distance between magnetic coil and rotating torus (see Fig.1).

-e electron charge $-1.602 \times 10-19 \mathrm{C}$.

$\hat{\boldsymbol{e}}_{z}$ unit vector in $\mathrm{z}$-direction.

$F_{\mathrm{e}}$ electrostatic force between 2 electrons.

$F_{\mathrm{g}}$ gravitational force between 2 electrons.

$F_{\mathrm{gp}}$ gravitophoton force, also termed HeimLorentz force, $\boldsymbol{F}_{g p}=\Lambda_{p} e \mu_{0} \boldsymbol{v}^{T} \times \boldsymbol{H}$, see Eq. (47). $G=G_{\mathrm{g}}+G_{\mathrm{gp}}+G_{\mathrm{q}}=6.67259 \times 10^{-11} \mathrm{~m}^{3} \mathrm{~kg}^{-1} \mathrm{~s}^{-2}$, gravitational constant.

$G_{\mathrm{g}}$ graviton constant, $G_{g} \approx G$ that is $G_{\mathrm{g}}$ decribes the gravitational interaction without the postulated gravitophoton and quintessence interactions.

$G_{\mathrm{gp}}$ gravitophoton constant, $G_{g p} \approx(1 / 67)^{2} G_{g}$.

$G_{\mathrm{q}}$ quintessence constant, $G_{q} \approx 4 \times 10^{-18} G_{g}$.

\section{$g_{i k}^{(g p)} \quad$ metric subtensor for the gravitophoton}

in subspace $I_{2} \cup S_{2}$ (see glossary for subspace description).

$\boldsymbol{g}_{i k}^{(p h)} \quad$ metric subtensor for the photon in subspace $I_{2} \cup S_{2} \cup T_{1}$ (see glossary for subspace description).

$h$ Planck constant $6.626076 \times 10^{-34} \mathrm{~J} \cdot \mathrm{s}$, $\hbar=h / 2 \pi$.

$h_{\mathrm{ik}}$ metric components for an almost flat spacetime.

$\ell_{\mathrm{p}}=\sqrt{\frac{G \hbar^{3}}{c^{3}}}=1.6 \times 10^{-35} \mathrm{~m}$ Planck length.

$m_{\mathrm{e}}$ electron mass $9.109390 \times 10^{-31} \mathrm{~kg}$. 
$m_{0}$ mass of proton or neutron $1.672623 \times 10^{-27} \mathrm{~kg}$ and $1.674929 \times 10^{-27} \mathrm{~kg}$.

$N_{\mathrm{n}}$ number of protons or neutrons in the universe.

q electric charge.

$R$ distance from center of coil to location of virtual electron in torus, see Fig.(1).

$r_{\mathrm{N}}$ distance from nucleus to virtual electron in torus, see Fig.(1).

$R_{-}$is a lower bound for gravitational structures, comparable to the Schwarzschild radius. The distance at which gravitation changes sign, $\rho$, is some 46 Mparsec.

$R_{+}$denotes an upper bound for gravitation and is some type of Hubble radius, but is not the radius of the universe, instead it is the radius of the optically observable universe. Gravitation is zero beyond the two bounds, that is, particles smaller than R- cannot generate gravitational interactions.

$r_{\mathrm{e}}$ classical electron radius

$$
r_{e}=\frac{1}{4 \pi \epsilon_{0}} \frac{e^{2}}{m_{e} c^{2}}=3 \times 10^{-15} \mathrm{~m} .
$$

$r_{\mathrm{ge}}$ ratio of gravitational and electrostatic forces between two electrons.

$v$ velocity vector of charges flowing in the magnetic coil, see Eq. (27), some $10^{3} \mathrm{~m} / \mathrm{s}$ in circumferential direction.

$v^{\mathrm{T}}$ bulk velocity vector for rigid rotating ring (torus) (see Sections. 3 and 4), some $10^{3} \mathrm{~m} / \mathrm{s}$ in circumferential direction.

$w_{\mathrm{gp}}$ probability amplitude (the square is the coupling coefficient) for the gravitophoton force (fifth fundamental interaction)

$$
w_{g p}^{2}=G_{g p} \frac{m_{e}^{2}}{\hbar c}=3.87 \times 10^{-49} \text { probability ampli- }
$$

tudes (or coupling amplitudes) can be distance dependent (indicated by a prime in [9]).

$w_{\text {gpe }}$ probability amplitude for emitting a gravitophoton by an electron

$$
w_{g p e}=w_{g p} .
$$

$w_{\mathrm{gpa}}$ probability amplitude for absorption of a gravitophoton by a proton or neutron

$$
w_{g p a}^{2}=G_{g p} m_{p} \frac{m_{e}}{\hbar c} .
$$

$w_{\mathrm{g} \_\mathrm{q}}$ conversion amplitude for the transformation of gravitophotons and gravitons into the quintessence particle, corresponding to the dark energy (rest mass of some $10^{-33} \mathrm{eV}$ ).

$w_{\mathrm{ph}}$ probability amplitude (the square is the coupling coefficient for the electromagnetic force, that is the fine structure constant $\alpha$ )

$$
w_{p h}^{2}=\frac{1}{4 \pi \epsilon_{0}} \frac{e^{2}}{\hbar c}=\frac{1}{137} \text {. }
$$

$w_{\text {ph_qp }}$ conversion amplitude for the transformation of photons into gravitophotons (see Eq. (35)).

$w_{\mathrm{q}}$ probability amplitude for the quintessence particle,(sixth fundamental interaction), corresponding to dark energy (rest mass of some $\left.10^{-33} \mathrm{eV}\right)$.

$\mathrm{Z}$ atomic number (number of protons in a nucleus and number of electrons in an atom)

$\mathrm{Z}_{0}$ impedance of free space,

$$
Z_{0}=\sqrt{\frac{\epsilon_{0}}{\mu_{0}}} \approx 376.7 \Omega .
$$

$\alpha$ coupling constant for the electromagnetic force or fine structure constant 1/137.

$\alpha_{\mathrm{gp}}$ coupling constant for the gravitophoton force .

Y ratio of probabilities for the electromagnetic and the gravitophoton force

$$
\gamma=\left(\frac{w_{p h}}{w_{g p}}\right)^{2}=1.87 \times 10^{46} .
$$

$\mu_{0}$ permeability of vacuum $4 \pi \times 10-7 \mathrm{~N} / \mathrm{m}^{2}$.

$\tau$ metron area (minimal surface $3 \mathrm{Gh} / 8 \mathrm{c}^{3}$ ), current value is $6.15 \times 10-70 \mathrm{~m}^{2}$.

$\Phi$ gravitational potential, $=\mathrm{GM} / \mathrm{R}$.

w rotation vector (see Fig. 1 ). 


\author{
Abbreviations \\ $B P P$ breakthrough propulsion physics \\ $G R$ General Relativity \\ HQT Heim Quantum Theory \\ LQT Loop Quantum Theory \\ LHS left hand side \\ ls light second \\ ly light year
}

QED Quantum Electro-Dynamics

RHS right hand side

$S R$ Special Relativity

VSL Varying Speed of Light

\section{Subscripts}

$e$ electron

gp gravitophoton

$g q$ from gravitons and gravitophotons into quintessence

$p h$ denoting the photon or electrodynamics

$s p$ space

\section{Superscripts}

em electromagnetic

gp gravitophoton

ph photon

$T$ indicates the rotating ring (torus)

Note: Since the discussion in this paper is on engineering problems, SI units (Volt, Ampere, Tesla or Weber $/ \mathrm{m}^{2}$ ) are used. $1 \mathrm{~T}=1 \mathrm{~Wb} / \mathrm{m}^{2}$ $=10^{4} \mathrm{G}=10^{4} \mathrm{Oe}$, where Gauss (applied to $\boldsymbol{B}$, the magnetic induction vector) and Oersted (applied to $\boldsymbol{H}$, magnetic field strength or magnetic intensity vector) are identical. Gauss and Oersted are used in the Gaussian system of units. In the MKS system, $\boldsymbol{B}$ is measured in Tesla, and $\boldsymbol{H}$ is measured in $\mathrm{A} / \mathrm{m}(1 \mathrm{~A} / \mathrm{m}=4 \pi$ $\left.\times 10^{-3} \mathrm{G}\right)$. Exact values of the physical constants are given in [22].
Note: For a conversion from CGS to SI units, the electric charge and magnetic field are replaced as follows:

$$
e \rightarrow e / \sqrt{4 \pi \epsilon_{0}} \text { and } \boldsymbol{H} \rightarrow \sqrt{4 \pi \mu_{0}} \boldsymbol{H} .
$$

\section{Space Propulsion and Higher-Di- mension Quantized Spacetime Phys- ics}

For effective and efficient lunar space transportation as well as interplanetary or even interstellar space flight a revolution in space propulsion technology is needed.

Regarding the requirements of NASA's Breakthrough Physics Propulsion Program (BPP) a revolutionary space propulsion system should

- use no or a very limited amount of fuel,

- possibility for superluminal speed, and

- requirement for a low energy budget. This immediately rules out any device flying close to the speed of light, since its mass is going to infinity, according to $S R$. A spacecraft having a mass of $10^{5} \mathrm{~kg}$, flying at a speed of $1 \%$ of the speed of light, carries an energy content of $4.5 \times 10^{17} \mathrm{~J}$. Even if the spacecraft can be provided with a $100 \mathrm{MW}$ nuclear reactor, it would take some 143 years to produce this amount of energy.

It is understood that the laws of current physics do not allow for such a revolutionary space propulsion system. Propulsion techniques of this type can only emerge from novel physics, i.e., physical theories that deliver a unification of physics that are consistent and founded an basic, generally accepted principles, either removing some of the limits, or giving rise to additional fundamental forces, and thus providing alternatives to current propulsion principles. Theories like $H Q T$ and $L Q T$ are therefore of great interest, since they might offer the potential for these advanced technologies, see, for instance, the remark on p. 9 in [29].

Hopes for such a unified theory are, indeed, not futile. In classical physics, science starts from the belief that space, time, and matter are infinitely divisible, in other words, that spacetime is continuous (a differentiable manifold in the mathematical sense) and not subjected to the quantization principle. Regarding the microcosm, there exists a large number of elementary particles that cannot be subdivided 
any further. In quantum physics arbitrary divisibility of matter has proved to be an illusion. On the other hand, the existence of matter is taken for granted, i.e., the occurrence of elementary particles is accepted as such, and the cause for the existence of matter cannot be revealed. There is substantial evidence that the currently favored Standard Model is far from being the final theory.

In the twentieth century there has been enormous progress in physics, based on both Einstein's theory of general relativity and quantum theory. Both theories are very successful in their own range, but could not be unified so far. The reason for the unification is ... Despite the successes of the two theories, the current status of physical theory lacks the understanding of the most fundamental physical facts. First, it has not been possible, despite numerous attempts over the last eight decades, to extent Einstein's idea beyond the range of gravitation. Second, $Q T$ has not been able to deliver the mass spectrum of elementary particles, nor is there a theoretical explanation for their lifetimes, neither can quantum numbers be derived. None of these theories is able to explain the nature of matter and inertia, topics that are essential for the physics of a completely novel propulsion system.

\subsection{Basic Concepts of HQT}

Einstein's view was eventually deemed untenable, because next to gravitation other forces became known. The recent article by L. Smolin [11] on Atoms of Space and Time, however, seems to be a sign that physics may be returning to the Einsteinian picture, namely the geometrization of the physical world, meaning that all forces (interactions) are ultimately determined by the structure of spacetime. The two important ingredients that Einstein did not use are a discrete spacetime and a higher-dimensional space, provided with special, additional features.

It is known that the general theory of relativity in a 4-dimensional spacetime delivers only one possible physical interaction, namely gravitation. Since Nature shows us that there exist additional interactions, and because both $G R$ and the quantum principle are experimentally verified, it seems logical to extend the geometrical principle to a discrete, higher-dimensional space. Consequently, Heim's quantum theory, $H Q T$, of gravity and elementary structures of matter is based on the geometric view of Einstein, namely that geometry itself is the cause of all physical interactions, but it uses the structure of Einstein's field equations only as a template for physical interactions in a higherdimensional discrete space, and extends them also to the microcosm.

Eventually developed by Heim and the first author, the theory utilizes an 8-dimensional discrete space 4 in which a smallest elemental surface, the so-called metron, exists. HQT, developed first by Heim in the fifties and sixties, and partly published in the following three decades of the last century, seems to be compliant with these modern requirements. It also makes a series of predictions with regard to cosmology and high energy physics [12] that eventually can be checked by experiment.

Most important, however, Heim's extended theory predicts two additional interactions [1, 6-9] identified as quintessence, a weak repulsive gravitational like interaction (dark energy) and gravitophoton interaction, that enables the conversion of electromagnetic radiation into a gravitational like field, represented by the two hypothetical gravitophoton (negative and positive energies) particles. The gravitophoton interaction is discussed in Chaps. [2, 3.1]. Quintessence (dark energy) is briefly discussed in Chap. [4].

The interpretation of the physical equations for the gravitophoton field leads to the conclusion that this field could be used to both accelerate a material body and to cause a transition of a material body into some kind of parallel space, possibly allowing superluminal speed. These effects could serve as the basis for advanced space propulsion technology, and are dealt with quantitatively in the following chapters.

4 To be more precise, Heim's theory was extended from 6 to 8 -dimensions by the first author and Heim, [7], to obtain the unification of the four known interactions (forces). In this process, it was found that two additional gravitational like interactions should occur, termed the gravitophoton field (attractive and repulsive) and the vacuum field (repulsive, interpreted later on as quintessence) $[1,7]$. The dimensional law derived by Heim requires a 12-dimensional space, but the additional four coordinates are needed only in the explanation of the steering of probability amplitudes (information). 
According to Heim's theory, gravitation, as we know it, is comprised of three interactions, namely by gravitons, the postulated gravitophotons, and by the quintessence particle. This means that the gravitational constant $G$ contains contributions from all three fields. The quintessence interaction, however, is much smaller than the first two contributions.

It is interesting to note, that the mass spectrum for elementary particles, calculated from Heim's mass formula, and partly shown in Appendix $A$ as taken from [12], is very sensitive to $G$. A corrected value of $G$ obtained by the first author, accounting for the contribution of the gravitophoton field, led to substantially improved results of the mass values when compared to experimental data. In Heim's theory the existence of matter as an independent entity is replaced by the features of a dynamic 8dimensional discrete space, and as such is created by space itself. In other words, matter is caused by a non-Euclidean metric in space $\mathbb{R}^{8}$, termed 8D Heim space, comprised by a large number of elemental space atoms (called metrons by Heim), interacting in a dynamic and highly complex way.

A few words about the history of $H Q T$ seem to be in place. Heim first published his theory of a higher-dimensional discrete spacetime in an obscure German journal [10] in a series of four articles in 1959. In 1977, following the advice of Heisenberg's successor, H.-P. Dürr, Heim published an article entitled Vorschlag eines Weges zur einheitlichen Beschreibung der Elementarteilchen (Recommendation of a Way to a Unified Description of Elementary Particles) [4], which in today's terminology was a summary of his theory for a unified field theory including quantum gravity. Later on, he wrote two text books Elementarstrukturen der $\mathrm{Ma}$ terie $[6,7]$ that were eventually published by A. Resch (see Acknowledgment). However, to be fair, it should be mentioned that Heim's publications are difficult to read, and needed to be modified and extended by the first author in several ways, for instance [9].

\subsection{LQT and HQT}

In order to understand how to categorize Heim's quantum theory, it seems worthwhile to determining the similarities between recent loop quantum theory by L. Smolin, A.
Ashtekar, and C. Rovelli [11, 24, 25] and $H Q T$, and also to learn how $H Q T$ compares with $G R$ and $Q T$. A major difference between $G R$ and Heim is that in $G R$ the material field source does not appear in geometrized form, but occurs as a phenomenological quantity (in the form of matter that is an entity of its own, whose existence is taken for granted).

It should be noted that $H Q T$ complements both $Q T$ and $G R$, in explaining the nature of elementary particles as well as their discrete mass spectrum and life times, based on the basis of a quantized geometrodynamics (quantized elemental areas of some $10-70 \mathrm{~m} 2$, termed metron by Heim) in a 12 dimensional space ( 3 real and 9 imaginary coordinates). As shown by Heim the fact that all additional coordinates are imaginary leads to real eigenvalues in the mass spectrum for elementary particles $[4,6]$. The idea of geometrization is extended to the sub-atomic range. However, in that case, the Christoffel symbols need to be replaced by real tensor components ${ }^{5}$. Heim derives a dimensional law that restricts the maximum number of dimensions to 12 and requires the existence of subspaces. From the metric of subspace $\mathbb{R}^{6}$, originally conceived by Heim, the premises of $Q T$ cannot be derived, and the quantization principle had to be introduced ad hoc. For instance, Dirac's equations cannot be derived within $\mathbb{R}^{6}$. However, when the metric in space $\mathbb{R}^{8}$ is considered, all possible physical interactions are reproduced. The complete space $\mathbb{R}^{12}$ is needed to explain how probability amplitudes (immaterial) are steering events in spacetime $\mathbb{R}^{4}$.

In the following, a Heim space is a quantized space comprising elemental surfaces with orientation (spin), the metron, whose size is the Planck length (apart from a factor) squared, comprising 6, 8, or 12 dimensions. A Heim space may comprise several subspaces, each equipped with its individual Riemannian metric. The union of these individual metric spaces is termed a poly-metric.

Furthermore, in GR the gravitational potential is associated with the metric tensor, and thus has a direct physical meaning. Extending this

5 This can be shown by employing the double transformation described in Eq. (2) to Heim's eigenvalue equations for the mass spectrum of elementary particles. Otherwise masses of particles could be transformed away which is unphysical. 
concept to the poly-metric in Heim space $\mathbb{R}^{8}$, and forming special combinations of these partial metrics, all possible fundamental physical interactions are obtained. Since GR has been extremely well verified experimentally, this interpretation seems to be justified.

\subsection{Fundamental Physical Interactions in 8-D Quantized Space}

In $G R$ the gravitational force is nothing but an effect of the geometric curvature of spacetime. The predictions of $G R$ have been tested extensively, and today $G R$ arguably is the experimentally best verified theory. Therefore, there is some confidence that this concept can be extended to all physical forces, and that the structure of the equations of $G R$ is valid for all physical interactions in a higher-dimensional space.

A Heim space $\mathbb{R}^{12}$, where the superscript denotes dimension, comprises five subspaces or partial structures that form semantic units. Combining these semantic units by employing certain selection rules a set of so called hermetry forms or partial metric tensors is obtained, forming the poly-metric, that represents all physical interactions [2]. Each of the semantic units (or subspaces) has its own metric. There are the subspaces $\mathbb{R}^{3}$ with real coordinates $\left(x_{1}\right.$, $\left.x_{2}, x_{3}\right)$, T1 with imaginary time coordinate $\left(x_{4}\right)$, $S^{2}$ with imaginary coordinates for organization of structures $\left(x_{5}, x_{6},\right), I^{2}$ with imaginary coordinates for information $\left(x_{7}, x_{8}\right)$, and $G^{4}$ with imaginary coordinates for steering of probality amplitudes and thus events in $\mathbb{R}^{4}\left(x_{9}, x_{10}, x_{11}\right.$, $\left.x_{12}\right)$. The space $\mathbb{R}^{12}$ is comprised of the two spaces $\mathbb{R}^{6}=\mathbb{R}^{3} \cup \mathrm{T}^{1} \cup \mathrm{S}^{2}$ and $\mathrm{V}^{6}=\mathrm{I}^{2} \cup \mathrm{G}^{4}$. The concept of energy exists in ${ }^{6} \mathbb{R}^{6}$, while $V^{6}$ is denoted as immaterial. Considering the space $\mathbb{R}^{8}=\mathbb{R}^{3} \cup T^{1} \cup S^{2} \cup I^{2}$, that is omitting the space $\mathrm{G}^{4}$, the theory predicts six fundamental interactions, instead of the four experimentally known ones. These interactions emerge in our spacetime and represent physical fields carrying energy. According to the theory, a transformation of electromagnetic energy into gravitational energy (gravitophoton) should be possible (see Chap. 2.5). It is this conversion that is used as the physical basis for the novel

6 This is not completely correct, since the vacuum or quintessence particles of hermetry form $\mathrm{H}_{10}\left(\mathrm{I}^{2}\right.$ ) (see glossary and [2]) with $\mathrm{I}^{2} \subset \mathbb{R}^{8}$ possess (small) energies. space propulsion concept $[1,2]$, which is not conceivable within the framework of current physics. This is a direct consequence of the dimension of Heim space, and the interpretation of a partial metric (hermetry form, see glossary) as a physical interaction or particle. In other words, if Einstein's view, namely of geometry being the cause of gravitation is extended to the poly-metric in Heim space, the interpretation of all physical interactions is a natural consequence. Moreover, the two additional interactions should also be considered real.

It seems that space $\mathrm{G}^{4}$ does not have a direct physical meaning in a sense that it is responsible for physical interactions. Its role seems to be acting as a symmetry breaking principle, responsible for a quantized bang, and much later on (some $10^{10}$ years ago), for the creation of matter.

Starting from Einstein's equations, Heim derives a set of nonlinear eigenvalue equations for microscopic particles (mass spectrum of elementary particles), first in $\mathbb{R}^{6}$. In Heim"s theory, quantum mechanics is not contained in $\mathbb{R}^{6}$, but in space $\mathbb{R}^{8}$. In this regard, Heim's theory can be understood as being complementary to the wave picture, taking care of the particle nature of physical objects (see [7] pp. 360 for the linear Dirac equations.

\section{The Physical Principles for Field Propulsion $^{7}$}

In the following a roadmap for the derivation of the gravitophoton interaction is presented.

The proposed propulsion concept works in two stages. First, a gravitophoton field is generated through interaction with an electromagnetic field that exerts a force on the space vehicle. Second, there exist parallel spaces in which covariant laws of physics are valid that allow speeds larger than the vacuum speed of light in $\mathbb{R}^{4}$. Under certain conditions, a spacecraft can enter a parallel space, see Sec. (3.3).

For the gravitophoton interaction to exist, Einstein's principle of geometrization of physics

7 The term breakthrough propulsion is not used, since it does not relate to the propulsion principle that is based on the conversion of photons (electromagnetic field) into gravitophotons (gravitational like field). 
needs to be valid for all physical interactions. According to $H Q T$ this is the case in $8 \mathrm{D}$ space.

For instance, in classical physics, there is no difficulty to include electromagnetism in general relativity by adding the stress-momentumtensor of the electromagnetic field $T_{i k}^{e m}$ to the RHS of Einstein's field equations in 4D spacetime

$$
R_{i k}=\kappa\left(T_{i k}^{g}+T_{i k}^{e m}-\frac{1}{2} g_{i k} T\right)
$$

and $T=T_{k}^{k}$ contains both the gravitational and electromagnetic contribution. The parameter $\mathrm{K}$ is of the form $\kappa=\frac{8 \pi G}{c^{4}}$. To complete the set of equations, Maxwell's equations have to be added. This is, however, not the approach of a unified field theory, because the electrodynamic field is added from the outside without any geometrical interpretation. In the next section, the concept underlying the unification of all physical interactions is derived, extending Einstein's principle of geometrization.

\subsection{The Physics of Hermetry Forms}

As described in [1] there is a general coordinate transformation $x_{m}\left(\xi_{\alpha}\left(\eta_{i}\right)\right)$ from

$$
\begin{gathered}
\mathbb{R}^{4} \rightarrow \mathbb{R}^{8} \rightarrow \mathbb{R}^{4} \text { resulting in the metric tensor } \\
g_{i k}=\frac{\partial x_{m}}{\partial \xi_{\alpha}} \frac{\partial \xi_{\alpha}}{\partial \eta_{i}} \frac{\partial x_{m}}{\partial \xi_{\beta}} \frac{\partial \xi_{\beta}}{\partial \eta_{k}}
\end{gathered}
$$

where indices $\alpha, \beta=1, \ldots, 8$ and $i, m, k=1, \ldots, 4$. The Einstein summation convention is used, that is, indices occurring twice are summed over. The above transformation is instrumental for the construction of the poly-metric used to describing physical interactions. The Euclidean coordinates $x_{\mathrm{m}}$ and curvilinear coordinates $\eta_{\mathrm{i}}$ are in $\mathbb{R}^{4}$, while curvilinear coordinates $\xi_{\alpha}$ are in $\mathbb{R}^{8}$. The metric tensor can be written in the form

$$
g_{i k}=: \sum_{\mu, v=1}^{8} g_{i k}^{(\mu \nu)}
$$

and the individual components are given by

$$
g_{i k}^{(\mu \nu)}=\frac{\partial x_{m}}{\partial \xi_{(\mu)}} \frac{\partial \xi_{(\mu)}}{\partial \eta_{i}} \frac{\partial x_{m}}{\partial \xi_{(v)}} \frac{\partial \xi_{(v)}}{\partial \eta_{k}} .
$$

Parentheses indicate that there is no index summation. In [2] it was shown that 12 hermetry forms (see glossary) can be established having direct physical meaning, involving specific combinations from the four subspaces. The following denotation for the metric describing hermetry form $H_{\ell}$ with $\ell=1, \ldots, 12$ is used:

$$
g_{i k}\left(H_{\ell}\right)=: \sum_{\mu, v \in H_{\ell}} g_{i k}^{(\mu \nu)}
$$

where summation indices are obtained from the definition of the hermetry forms. The expressions $g_{i k}\left(H_{\ell}\right)$ are interpreted as different physical interaction potentials caused by hermetry form $H_{\ell}$, following the interpretation employed in $G R$. The combination of coordinates $\xi_{\mu}$ and $\xi_{v}$ are characteristic for the interaction, and also characterize the subspace. Applying the sieve operator formed from Kronecker symbols, namely

$$
s\left(\mu_{0}, v_{0}\right):=\delta_{\mu_{0} \mu} \delta_{v_{0} v}
$$

to Eq. (5) selects the term $g_{i k}^{\left(\mu_{0} v_{0}\right)}$. A sieve operator (or sieve transformation) can be applied repeatedly, and thus serves to convert one hermetry form into another one. At the moment a sieve operator is a mathematical construction only, but it is the aim of this discussion to show how such a conversion can be obtained in physical reality. For the sake of simplicity, the following short form, omitting subscripts $i k$, is introduced $(\mu \nu):=g_{i k}^{(\mu v)}$.

Next the hermetry forms pertaining to the three subspaces $\mathrm{S}^{2}, \mathrm{I}^{2}, \mathrm{~S}^{2} \times \mathrm{I}^{2}$ are investigated. Cosmological data clearly show that the universe is expanding, which indicates a repulsive interaction. Gravitational attraction is well known since Newton. Both interactions act on matter, so that there should be two hermetry forms having anti-symmetric properties. The spaces corresponding to these interaction are identified as $\mathrm{S}^{2}$ and $\mathrm{I}^{2}$. The gravitational field, as described by gravitons, is given by hermetry form $\mathrm{H}_{12}$ 


$$
g_{i k}\left(H_{12}\right)=(55)+(56)+(65)+(66),
$$

while the vacuum field (quintessence) is given by

$$
g_{i k}\left(H_{10}\right)=(77)+(78)+(87)+(88) .
$$

There is a third hermetry form whose metric is in the space $\mathrm{S}^{2} \times \mathrm{I}^{2}$. Since this metric is a combination of an attractive and a repulsive interaction, it is assumed that there are exist two fields. The first partial metric is considered to be attractive, since its components contain the gravitational metric of Eq. (7). For the same reason the second part is considered to be repulsive. The particle for mediating this interaction is called the gravitophoton because of the possible interaction with the electromagnetic field. The reasons will become clear in the next section. It is postulated from the metrics of Eqs. $(9,10)$ that there are two types of gravitophotons associated with the attractive and the repulsive gravitophoton potentials. Their respective coupling constants are denoted by

$G_{g p}^{-}$and $G_{g p}^{+}$that will be described below. The attractive gravitophoton particle is described by Eq. (9), the minus sign denoting negative energy density, because it contains the metric of the graviton which is directly visible from Eq. (7). The repulsive gravitophoton particle is described by Eq. (10), the plus sign denoting positive energy density, because it contains the metric of the vacuum or quintessence particle that describes a repulsive force. Their partial metric have the form

$$
\begin{aligned}
g_{i k}\left(H_{11}^{-}\right)= & (55)+(56)+(65)+(66)+ \\
& (57)+(67)+(58)+(68) \\
g_{i k}\left(H_{11}^{+}\right)= & (77)+(78)+(87)+(88)+ \\
& (75)+(76)+(85)+(86) .
\end{aligned}
$$

To conclude this section, it has been shown that in Heim space $\mathbb{R}^{8}$ there are three physical interactions acting on material particles, namely, gravitation represented by hermetry form $\mathrm{H}_{12}\left(\mathrm{~S}^{2}\right)$ (attractive), the quintessence or vacuum field hermetry form $\mathrm{H}_{10}\left(\mathrm{I}^{2}\right)$ repulsive), and the gravitophoton field, hermetry form $\mathrm{H}_{11}$ $\left(\mathrm{S}^{2}, \mathrm{I}^{2}\right)$ (both attractive and repulsive). Negative and positive gravitophotons are generated simultaneously in pairs, and $\mathrm{H}_{11}$ is the only hermetry form that is identically 0 , that is

$$
g_{i k}\left(H_{11}\right)=g_{i k}\left(S^{2} \times I^{2}\right)=0 .
$$

It is a strange fact that a hermetry form that is zero should have any physical effect at all. This reflects the fact that the total energy being extracted from the vacuum by pair production of gravitophotons is zero. However, the physical effect lies in the different absorption coefficients of negative and positive gravitophotons. As it turns out in Chap. 3, gravitophotons are generated by virtual electrons, that is, they are generated by vacuum polarization ${ }^{8}$. In this process energy is conserved, but two different types of energy both negative and positive are obtained, adding up to zero. $\mathrm{H}_{11}$ is the only hermetry form that is comprised by space $\mathrm{S}^{2} \times \mathrm{I}^{2}$, the so called transcoordinates ${ }^{9}$. No other of the hermetry forms is identical to 0 , since this is the only hermetry form associated with creating particles from the vacuum.

Hence, the gravitational constant $G$ is comprised of the three individual coupling strengths of these interactions

$$
G=G_{g}+G_{g p}+G_{q}
$$

where $^{10}$

$$
G_{g p} \approx 1 / 67^{2} G_{g} \text { and } G_{q} \approx 4 \times 10^{-18} G_{g} .
$$

In the following section the metric describing photons, given by hermetry form $\mathrm{H}_{5}\left(\mathrm{~T}^{1}, \mathrm{~S}^{2}\right.$, $\mathrm{I}^{2}$ ), and its interaction with the gravitophoton metric is investigated.

8 A nonzero vacuum density during the early universe resulted in an exponential expansion (inflationary phase), and also is the cause of the Casimir effect, although extremely small. $G R$ alone cannot provide the physics for field propulsion. Moreover, vacuum energy is also considered to be responsible for the expansion of the universe.

9 Coordinates of $\mathrm{S}^{2}$ are associated with GR and those of $\mathrm{I}^{2}$ are assigned to QT.

10 In the physical interaction picture, generally the first partner emits and the second one absorbs a messenger particle. Since the quintessence is formed from the vacuum itself, there is no generating mass, for instance, a proton mass emitting a photon. Thus the value $G_{q}$ is some kind of fictitious value. 


\subsection{The Metric for Electromagnetic Interac- tions}

The metric tensor for photons depends on subspaces $\mathrm{I}^{2}, \mathrm{~S}^{2}$, and $\mathrm{T}^{1}$ with coordinates $\xi_{4}$, $\xi_{5}, \ldots, \xi_{8}$, see [2].

$$
g_{i k}^{(p h)}:=g_{i k}\left(H_{5}\right)=\sum_{\mu, v=4}^{8} g_{i k}^{(\mu v)}
$$

The coupling constant of this hermetry form is

$$
\alpha=w_{p h}^{2}=\frac{1}{4 \pi \epsilon_{0}} \frac{e^{2}}{\hbar c} \text {. }
$$

For weak electrodynamic and also for weak gravitational fields, spacetime (4D) is almost flat, so one obtains

$g_{i k}=g_{i k}^{(0)}+h_{i k} \quad$ where $\quad g_{44}^{(0)}=-1 \quad$ and $g_{i i}^{(0)}=+1$ where $i=1,2,3$ and $k, \ell=1, \ldots, 4$. The hik are small quantities whose products are negligible. In the following the $h_{\mathrm{ik}}$ are used to describe the metric for electromagnetic interactions. All other components are 0. The geodesic equation [1] takes the form

$$
\ddot{x}^{i}=-\frac{1}{2}\left(\frac{\partial h_{i l}}{\partial \eta_{k}}-\frac{\partial h_{k l}}{\partial \eta_{i}}+\frac{\partial h_{i k}}{\partial \eta_{l}}\right) \dot{x}^{k} \dot{x}^{l} .
$$

where the dot denotes the time derivative. Evaluating the terms on the RHS gives

$$
\begin{aligned}
-2 \ddot{x}^{i}= & \left(2 h_{i 4,4}-h_{44, i}\right) \dot{x}^{4} \dot{x}^{4}+ \\
& 2\left(h_{i 4, l}+h_{i l, 4}-h_{4 l, i}\right) \dot{x}^{4} \dot{x}^{l}+ \\
& \left(h_{i k, l}+h_{i l, k}-h_{k l, i}\right) \dot{x}^{k} \dot{x}^{l} .
\end{aligned}
$$

with $i, k, \ell=1,2,3$ and the comma denoting a partial derivative. Investigating the first two terms of Eq. (14) and using $x^{4}=c t \quad{ }^{11}$ one obtains

$$
\ddot{x}^{i}=\left(\frac{1}{2} h_{44, i}-h_{i 4,4}\right) c^{2}+\left(h_{4 l, i}-h_{i 4, l}\right) c \dot{x}^{l} .
$$

Introducing the quantity

$$
M_{i k}=\left(\frac{1}{1+\delta_{k 4}} h_{k 4, i}-h_{i 4, k}\right) \text {, }
$$

Eq. (15) can be written in shortform

11 It should be noted that in this section contravariant coordinates are used.

$$
\ddot{x}^{i}=M_{i k} c \dot{x}^{k} .
$$

This form can be directly compared with an electron moving in an electromagnetic field

$$
\ddot{x}^{i}=\frac{e}{m_{e} c} F_{i k} \dot{x}^{k}
$$

where $\quad F_{i k}=\frac{\partial A_{i}}{\partial x^{k}}-\frac{\partial A_{k}}{\partial x^{i}}$ and no distinction needs to be made between covariant and the ordinary derivative. The electromagnetic field tensor is obtained from the 4-vector electromagnetic potential that is defined as $\left(\Phi, A_{i}\right)$. From comparison of Eqs. (16) and (17) the following expression for the metric is obtained

$$
h_{4 k}=\frac{e}{m_{e} c^{2}}\left(1+\delta_{4 k}\right) A_{k} .
$$

In the next step, the third term of Eq. (14) is investigated. Tensor potentials $h_{i k}$ can be written, see [1], by means of retarded potentials

$$
h_{i k}=\frac{1}{4 \pi \epsilon_{0}} \frac{e Q}{m_{e} c^{2} r} \frac{v_{i}}{c} \frac{v_{k}}{c}=\frac{e}{m_{e} c^{2}} A_{i} \frac{\dot{x}^{k}}{c}
$$

with $i, k=1,2,3$. Combining Eqs. (17) and (18) with Eq. (19), one obtains

$$
h_{i k}=\frac{1}{4 \pi \epsilon_{0}}\left(1+\delta_{4 i} \delta_{4 k}\right) \frac{e Q}{m_{e} c^{2} r} \frac{v_{i}}{c} \frac{v_{k}}{c}
$$

with $i, k=1, \ldots, 4$.

Analyzing Eq. (20) shows that for $i=4$ and $k=4$ the metric describes the electric potential $\Phi$, while for $k=4$ and $i=1,2,3$ the metric represents the vector potential $\boldsymbol{A}$. For indices $i, k=1,2,3$ an additional tensor potential is obtained, which is not present in classical electrodynamics. Therefore, a $4 \times 4$ matrix is needed to describe all electromagnetic potentials.

Tensor potentials $h_{i k}$ with $i, k=1,2,3$ are belonging to the hermetry form for photons and thus have coupling coefficient $\alpha=w_{p h}^{2}$, but cannot be associated with the 4-potential of the electromagnetic field. Introducing the coupling coefficient $\alpha$ in Eq. (20) leads to 
$h_{i k}=\alpha\left(1+\delta_{4 i} \delta_{4 k}\right) \frac{Q}{e} \frac{\hbar}{m_{e} c} \frac{1}{r} \frac{v_{i}}{c} \frac{v_{k}}{c}$.

On the other hand, $h_{i k}=\sum_{\mu, v=4}^{8} h_{i k}^{(\mu \nu)}$ is defined

by its sum of partial potentials, so that the sum of all of these potentials is determining the coupling constant for the electromagnetic field, namely $w_{\text {ph. }}$. Coupling constants for different fields are thus determined by the corresponding sum of their partial potentials.

\subsection{The Metric for Coupling Electromagne- tism and Gravitation}

As was shown above, the metric tensor for the gravitophoton depends on subspaces $\mathrm{S}^{2}$ and $\mathrm{I}^{2}$ with coordinates $\xi_{5}, \xi_{6}, \xi_{7}, \xi_{8}$, and is written as

$$
g_{i k}^{(g p)}:=g_{i k}\left(H_{11}\right)=\sum_{\alpha, \beta=5}^{8} g_{i k}^{(\alpha \beta)}=0 \text {. }
$$

In comparison with Eq. (8), the metric for the photon can be written in the form ${ }^{12}$

$$
g_{i k}^{(p h)}=g_{i k}^{(g p)}+g_{i k}^{(44)}+\sum_{\alpha, \beta=5}^{8}\left(g_{i k}^{(\alpha 4)}+g_{i k}^{(4 \beta)}\right) .
$$

The second and third terms, as will be shown below, can be associated with the electric force (electric scalar potential) and the Lorentz force (vector potential). The first term represents the combined metric for the negative and positive gravitophoton particles. If an experiment can be conceived which causes the metric of the photon to become 0 , then the metric for the gravitophoton particles remains. The experiment needs to remove the time dependence from the photon metric, so that only the space $\mathrm{S}^{2} \times \mathrm{I}^{2}$ remains, responsible for the gravitophoton metric ${ }^{13}$.

In addition it can be shown, see Eq. (35), that in the presence of virtual electrons, responsible for the vacuum polarization and the shielding of the charge of a nucleus [16], there exists a

12 The sum of the second and third terms were denoted as electromagnetic metric tensor, $g_{i k}^{(e m)}$, in [1].

13 We are aware of the fact that these theoretical predictions sound highly speculative, but they are direct consequence of the geometrization principle. nonzero probability amplitude for converting a photon into gravitophotons. This gravitational force is the basis for the propulsion concept, termed gravitophoton field propulsion or field propulsion.

For weak gravitational fields, spacetime is almost flat, so the contribution of $\frac{\partial \xi_{4}}{\partial \eta_{4}}$ is large in comparison to $\frac{\partial \xi_{4}}{\partial \eta_{l}}, l=1,2,3$. Therefore, only the scalar photon potential needs to be considered

$$
g_{44}^{(p h)}=g_{44}^{(0)}+h_{44}^{(p h)} .
$$

For the linearized potential a formula similar to Eq. (23) holds,

$$
\begin{gathered}
h_{44}^{(p h)}=h_{44}^{(44)}+\sum_{\alpha, \beta=5}^{8}\left(h_{44}^{(\alpha 4)}+h_{i k}^{(4 \beta)}\right) \\
+\sum_{\alpha, \beta=5}^{8} h_{44}^{(\alpha \beta)} .
\end{gathered}
$$

Next, the contributions of the partial potentials on the RHS of Eq. (25) are evaluated. From the known form of the electric and Lorentz forces, $\quad \boldsymbol{F}=q \boldsymbol{E}+q \boldsymbol{v}^{T} \times \boldsymbol{B}, \quad v^{\mathrm{T}}$ denoting the velocity of the rotating torus, there follows the existence of a scalar electric potential $\phi$ and a vector potential $A$ with components $A_{i}=\mu_{0} \frac{Q v_{i}}{R}$ where Qvi denotes the total cur-

rent in the magnetic coil and $i=1,2,3$. The first term in (25) is associated with the electric potential, seen by a virtual electron with charge $-e$ at distance $r_{\mathrm{N}}$ from a nucleus, located in the torus, see Fig. (1). The potential thus takes the form

$$
h_{44}^{(44)}=-\frac{1}{4 \pi \epsilon_{0}} \frac{1}{m_{e} c^{2}} \frac{e Z e}{r_{N}} .
$$

For the first stage of the proposed propulsion mechanism as well as for the experiment of Fig. (1), it can be assumed that speed $v_{\mathrm{i}}<<c$. No field propulsion system will accelerate a spacecraft to a velocity comparable to the speed of light, since the required energy renders such an approach impractical. 
The second partial potential can be determined from Eq. (19). The corresponding vector potential is of the form

$$
\sum_{\alpha, \beta=5}^{8}\left(h_{44}^{(\alpha 4)}+h_{44}^{(4 \beta)}\right)=-\frac{1}{4 \pi \epsilon_{0}} \frac{1}{m_{e} c^{2}} \frac{e Q}{R} \frac{v_{i}}{c} \frac{v_{i}^{T}}{c}
$$

with summation over $i=1,2,3$ and $v_{\mathrm{i}} / c$ and $Q$ the speed and total charge of the electrons in the current loop (see Fig. (1)), while $v_{i}^{T} / c$ denotes a velocity component of the rotating torus (see Fig. (1)). The charge $-e$ denotes electron charge. $R$ is the distance from the center of the coil to the location of a virtual electron in the torus. This potential represents the Lorentz force.

There is a third partial potential in Eq. (25) that has the form of a tensor potential, which has no counterpart in classical electrodynamics theory and comes from the geodesic equation. According to the geometrization of forces, a new force should exist, derived from

$$
\begin{aligned}
& \sum_{\alpha, \beta=5}^{8}\left(h_{44}^{(\alpha \beta)}\right)= \\
& -\frac{1}{4 \pi \epsilon_{0}} \frac{1}{m_{e} c^{2}} \frac{e Q}{R} \frac{v_{i}}{c} \frac{v_{i}^{T}}{c}\left(\frac{v_{k}}{c} \frac{v_{k}^{T}}{c}\right)
\end{aligned}
$$

with summation over $i$ and $k$, assuming values $1,2,3$. The potential of Eq. (28) describes a scalar potential that exists at a location in space carrying a specific charge $e / m_{\text {e }}$. Adding up all three contributions results in a potential

$$
\begin{aligned}
& h_{44}^{(p h)}=\frac{1}{4 \pi \epsilon_{0}} \frac{1}{m_{e} c^{2}} \times \\
& \left(\frac{e Z(r) e}{r_{N}}-\frac{e Q}{R} \frac{v_{i}}{c} \frac{v_{i}^{T}}{c}-\frac{e Q}{R} \frac{v_{i}}{c} \frac{v_{i}^{T}}{c} \frac{v_{k}}{c} \frac{v_{k}^{T}}{c}\right) .
\end{aligned}
$$

For distances $r<r_{\mathrm{N}}, Z(r)$ is replacing $Z$, accounting for the shielding effect of the charge of the nucleus by the virtual electrons that are being formed in the vicinity of a nucleus within the range of the Compton wavelength of the electron. It should be noted that the electron charge, $-e$, was used in the first term. In the second and third terms it should be noted that $e Q>0$, since electrons are involved. From Eq. (27) it is required that the 4-dimensional vector potential, $\left(\phi, A_{\mathrm{i}}\right)$ with $i=1,2,3$, of classical electrodynamics has to be replaced by the 4-dimensional tensor potential $\left(\phi, A_{\mathrm{i}}, A_{\mathrm{ik}}\right)$ with $i, k=1,2,3$. Since velocities of charges in a material body are much smaller than the speed of light, the value of the factor $v_{k} / c v_{k}^{T} / c$ being in the range of $10^{-11}$ to $10^{-16}$, it is understandable that the tensor potential was not separately identified so far. Expressing

$$
e Z(r) e=e Z e+e Z \Delta e(r) \text { where } \Delta e(r) \text { rep- }
$$

resents the additional positive charge of the nucleus resulting from the shielding effect of the virtual electrons (see below), Eq. (29) takes the form

$$
\begin{gathered}
h_{44}^{(p h)}=\frac{1}{4 \pi \epsilon_{0}} \frac{1}{m_{e} c^{2}} \times \\
\left(\frac{e Z e}{r_{N}}+\frac{e Z \Delta e}{r_{N}}-\frac{e Q}{R} \frac{v_{i}}{c} \frac{v_{i}^{T}}{c}-\frac{e Q}{R} \frac{v_{i}}{c} \frac{v_{i}^{T}}{c} \frac{v_{k}}{c} \frac{v_{k}^{T}}{c}\right) .
\end{gathered}
$$

Considering a nucleus of one of the atoms in the material comprising the torus, there is a location $r_{\mathrm{N}}$ for which the first and third terms of Eq. (30) cancel, namely for

$$
r_{N}=\frac{Z e}{Q} R \frac{c}{v_{i}} \frac{c}{v_{i}^{T}}
$$

where the constant charge value $\mathrm{Ze}$ was used. With $\Delta e=A e$, see Eq. (35), the following equation holds

$$
\frac{e Z \Delta e}{r_{N}}=\frac{A e Q}{R} \frac{v_{i}}{c} \frac{v_{i}^{T}}{c} .
$$

The value of $A$, derived from vacuum polarization, is specified in Eq. (36) and computed in Eqs. $(37,38)$. If the value $r_{\mathrm{N}}$ is smaller than

$$
\lambda_{C}=\frac{h}{m_{e} c}=2.43 \times 10^{-12} \mathrm{~m} \text {, the Compton wave- }
$$

length of the electron, the second term in (30) is different from 0 and the speed $v_{\mathrm{i}}$ can be chosen such that the first and the third terms cancel, leading to

$$
h_{44}^{(p h)}=\frac{1}{4 \pi \epsilon_{0}} \frac{1}{m_{e} c^{2}} \frac{e Q}{R} \frac{v_{i}}{c} \frac{v_{i}^{T}}{c}\left(A-\frac{v_{k}}{c} \frac{v_{k}^{T}}{c}\right) .
$$

From the nature of $A$, it is obvious that the first term in the above potential is generated from the vacuum, wile the second term comes from the tensor potential generated in the coil. The total energy extracted from the vacuum is, however, always zero. According to L. Krauss in [3] the cosmological constant is $5 \times 10^{-10}$ $\mathrm{J} / \mathrm{m}^{3}$. This means that the conversion of photons into gravitophotons begins to occur as soon as the condition $h_{44}^{(p h)} \approx 0$ is satisfied. 


\subsection{Physical Model for Gravitophoton Gen- eration}

In the following, starting from Eq. (33), the physical mechanism is presented, responsible for the conversion of photons into gravitophotons. The mechanism for the generation of the postulated negative and positive gravitophoton particles is based on the concept of vacuum polarization known from Quantum Electrodynamics $(Q E D)$. In $Q E D$ the vacuum behaves like a dielectric absorbing and producing virtual particles and the Coulomb potential is associated with the transfer of a single virtual photon. Vacuum polarization in form of the electron-photon interaction changes the Coulomb potential of a point charge for distances within the electron Compton wavelength with respect to a nucleus.

The velocities $v_{i}, v_{i}^{T}$ in combination with the total charge $Q$ in the current loop or magnetic coil need to be chosen such that

$$
r_{N}<\lambda_{C},
$$

otherwise vacuum polarization does not occur. It should be noted that the experiment allows to vary these three parameters. However, as will be shown below, two more conditions have to be satisfied. In addition, the material in the torus should contain hydrogen atoms to get a value of $Z$ as small as possible, that is close to 1 .

A conversion of photons into gravitophotons is possible according to Eqs. (35). The first equation describes the production of $N^{2}$ gravitophoton particles ${ }^{14}$ from photons. This equation is obtained from Heim's theory in 8D space in combination with considerations from number theory, and predicts the conversion of photons into gravitophoton particles. The second equation is taken from Landau [16]

$$
\begin{aligned}
& w_{p h}(r)-w_{p h}=N w_{g p} \\
& w_{p h}(r)-w_{p h}=A w_{p h} .
\end{aligned}
$$

The physical meaning of Eqs. (35) is that an electromagnetic potential containing probability amplitude $A w_{\mathrm{ph}}$ can be converted into a

14 The factor $N^{2}$ results from the fact that in Eq. (35) probability amplitudes are considered, but the generation of particles depends on actual probabilities. It should be noted that $N$ is not needed, but the product $N w_{g p}$. gravitophoton potential with associated probability amplitude $N w_{\text {gp }}$. From Eqs. (35) the following relation holds for gravitophoton production, requiring the existence of a shielding potential

$$
N w_{g p}=A w_{p h} .
$$

The function $\mathrm{A}(\mathrm{r})$ can be calculated from Landau's radiation correction [16] and is given by

$$
A=\frac{2}{3 \pi} \alpha \int_{1}^{\infty} e^{-2 \frac{m_{e} c}{\hbar} r \xi}\left(1+\frac{1}{2 \xi^{2}}\right)\left(\xi^{2}-1\right)^{1 / 2} / \xi^{2} \quad \text { (3) }
$$

with numerical values for $A$ ranging from $10^{-3}$ to $10^{-4}$. For small $r\left(r<<\lambda_{\mathrm{C}}\right)$ the integral in Eq. (37) can be evaluated

$$
A=-\frac{2 \alpha}{3 \pi}\left(\ln \frac{m_{e} c}{\hbar} r+C_{E}+\frac{5}{6}\right)
$$

where $C_{\mathrm{E}}=0.577$ is Euler's constant. For $r>$ $\lambda_{C}$, the integral Eq. (37) falls off exponentially as $e^{-2 \frac{m_{e} \hbar}{c} r}$. Vacuum polarization changes the Coulomb potential of a point charge only for distances $r<\lambda_{\mathrm{c}}$. The radiation correction is not only caused by electron-positron interaction, but interaction with muons and pions is also possible. $Q E D$ works for muons, but does not work for pions, since they are subject to the strong interaction. Therefore, for $r \sim h / \mathrm{m}_{\pi} c$, $Q E D$ will not suffice anymore, i.e., there is no applicable theory. Hence, the physical model presented below is limited to this fact.

The third condition is, according to Eq. (33), to make the photon potential vanish, i.e., to trigger the conversion of a photon into negative and positive gravitophotons, which requires that $A$ takes on a value $\tilde{A}$ that is

$$
\tilde{A}=\frac{v_{k}}{c} \frac{v_{k}^{T}}{c}
$$

where the value of $\tilde{A}$ depends on the velocities of the charges in the coil and the rotating torus. This conversion takes place at a larger value of $r$, since the product on the RHS of Eq. (39) is some $10^{-11}$.

\subsection{Conversion of Photons into Gravitopho- tons}

To summarize, there are the following three conditions to be satisfied in order to convert a photon into a pair of negative and positive 
gravitophotons while insuring that the total energy extracted in form of gravitophoton particles from the vacuum is zero.

$$
\begin{gathered}
\tilde{A}=\frac{v_{k}}{c} \frac{v_{k}^{T}}{c} \\
r_{N}<\lambda_{C}=\frac{h}{m_{e} c} \\
r_{N}=\frac{Z e}{Q} R \frac{c}{v_{i}} \frac{c}{v_{i}^{T}}
\end{gathered}
$$

The crucial point in the interpretation of Eq. (40) is that the first equation provides a value of $\tilde{A} \approx 10^{-11}$. This value is needed to start converting photons into gravitophotons. However, for this value of $\tilde{A}$ the conversion process is not efficient, i.e., the number of gravitophotons produced is too small to result in an appreciable force. Equations two and three determine the conditions at which, according to Eq. (42), an effective gravitophoton potential exists for which the respective value $r_{\mathrm{N}}$ is determined. The corresponding value for $A>\tilde{A}$ is some $10^{-3}$. It should be noted that Eq. (39) is not interpreted as a resonance phenomenon, but sets a condition for the photon potential to disappear and the gravitophoton potential to appear that is, for the onset of the conversion of photons into gravitophotons. Once this happened, the value of $A$ can be increased further, giving rise to an efficient and effective gravitophoton potential for field propulsion ${ }^{15}$.

In the following these conditions will be employed to determining the technical requirements of a gravitophoton propulsion device. Since an almost flat space was assumed, the equation for the gravitophoton metric, Eq. (22), is reduced to a single component in direct analogy to Eq. (25), and thus the equation for the gravitophoton potential can be written as

$$
h_{44}^{(g p)}=\frac{1}{4 \pi \epsilon_{0}} \frac{1}{m_{e} c^{2}} \frac{e Q}{R} \frac{v_{i}}{c} \frac{v_{i}^{T}}{c} A \text {. }
$$

From the nature of $A$, it is obvious that the above potential is generated from the vacuum. In addition, a factor $\frac{N^{\prime} w_{g p}}{w_{p h}}=1$ is introduced in Eq. (42) to emphasize that this equation does not contain any electrical charges any-

15 It should be noted that this not a proof that the conversion process takes place as indicated. Only the experiment can prove the correctness of this assumption. more, since it describes a purely gravitational field.

Replacing $A$ by Eq. (36) and insuring that the potential of Eq. (41) identically vanishes, the converted gravitophoton field takes the form

$$
h_{44}^{(g p) \mp}=\mp\left(\frac{N w_{g p}}{w_{p h}}\right)\left(\frac{N^{\prime} w_{g p}}{w_{p h}}\right) \frac{1}{4 \pi \epsilon_{0}} \frac{1}{m_{e} c^{2}} \frac{e Q}{R} \frac{v_{i}}{c} \frac{v_{i}^{T}}{c}
$$

The $\mp$ sign in Eq. (42) represents the fact that there are both attractive and repulsive gravitophotons as described by the two metric forms in Eqs. $(9,10)$. The sum of the two potentials adds up to 0, satisfying Eq. (22). The gravitophoton field is a gravitational like field, acting on material particles, except that it can be both attractive and repulsive, and is represented by two different types of gravitophotons. However, the coupling constants of the two particles are different, and only the negative (attractive) gravitophotons are absorbed by protons and neutrons, while absorption by electrons can be neglected ${ }^{16}$. Under certain circumstances, a material body may be able to transition into a postulated parallel space that is not subject to the limit $c$, the vacuum speed of light. Any gravitophoton propulsion device therefore works as a two-stage system, first accelerating the spacecraft by gravitophoton force and then, for certain values of the magnetic field and torus properties, causes a transition into parallel space.

\section{Space Flight Dynamics of Gravito- photon Field Propulsion}

In this chapter the two-stage gravitophoton propulsion system is discussed. In Secs. (3.1, 3.2) the acceleration phase is described, and Sec. (3.3) discusses the transition into parallel space, presenting the physical laws governing parallel space. In addition, the physical conditions for transition into and leaving parallel space are outlined.

\subsection{Gravitophoton Interaction Equations for Space Propulsion}

Negative gravitophotons are subsequently absorbed by the protons in the torus which have a large absorption cross section compared to positive gravitophotons. In the non-relativistic case, the scattering cross section for photon-

16 The amount of energy extracted from the vacuum in gravitophoton pair production is zero. 


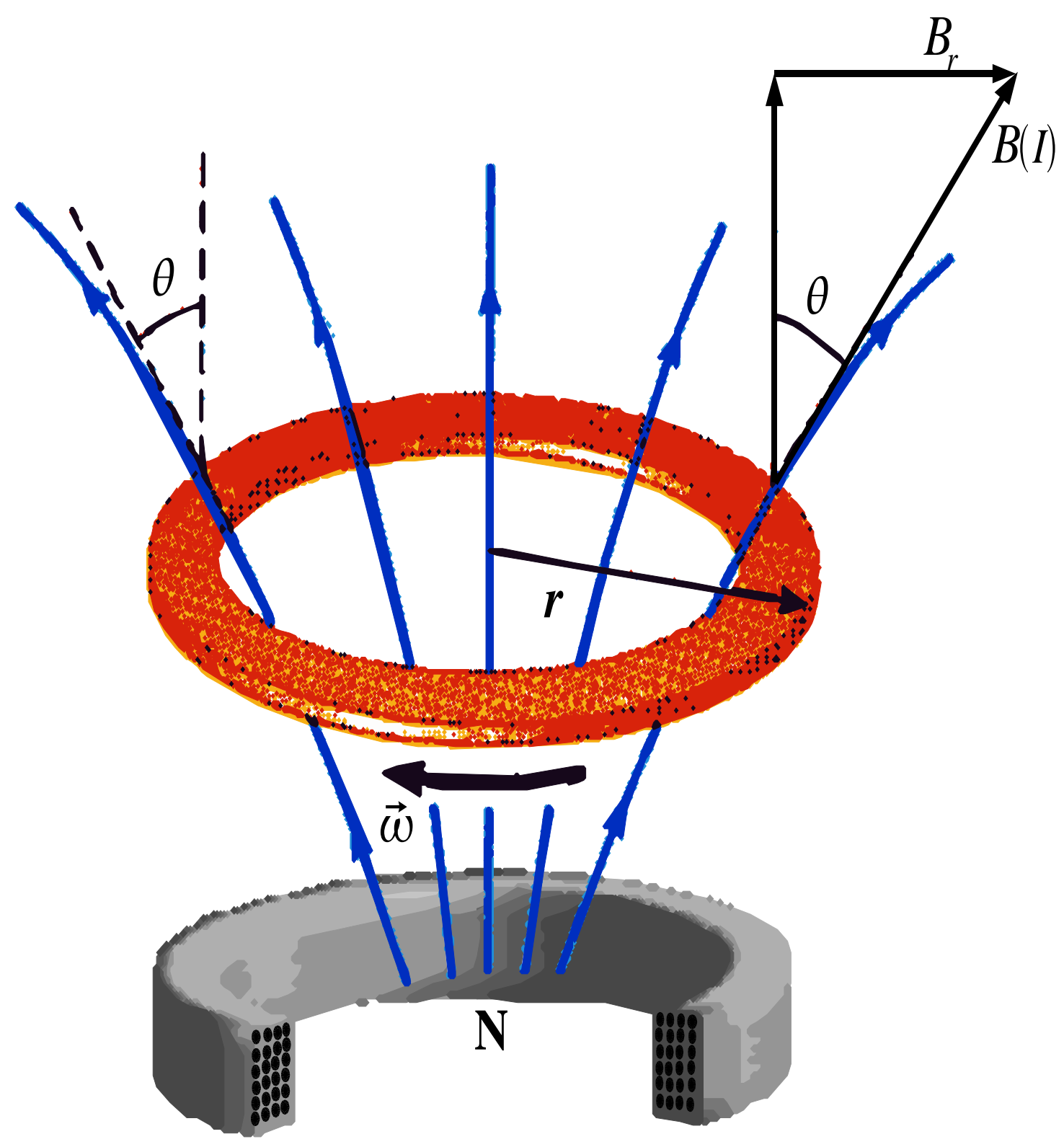

Figure 1: Instead of a simple current loop, a coil with many turns can be used. Both, the current in the coil and the rotation are in counter-clockwise direction. The field of this coil gives rise to an inhomogeneous magnetic field that has a radial field component. The radial component and the gradient in $\mathrm{z}$-direction are related through $H_{r}=-\frac{r}{2} \frac{\partial H_{z}}{\partial z}$. It should be noted, however, that if the ring possesses a magnetic moment, M, there is a magnetic force in the z-direction of magnitude $F=M \frac{\partial H_{z}}{\partial z}$. This force does not depend on the rotation of the ring. For a diamagnetic material the force acts in the positive z-direction (up), while para- and ferromagnetic materials are drawn toward the region of increasing magnetic field strength (down). The gravitophoton fore superimposes these effects.

The gravitophoton force comes into play as soon as the ring starts rotating and the condition according to Eq. (40) is satisfied. i.e., the velocity components $v_{k}$ and $v_{k}^{T}$ must have the same sign. Perhaps equipment used to measuring magnetic moments can be employed to determine the gravitophoton force. For instance, if a paramagnetic substance is used, the gravitophoton force (up) could be used to balance the magnetic force, so that the resulting force is 0 . From Refs. [23 and 24] it is found that a quartz sample $\left(\mathrm{SiO}_{2}\right.$, diamagnetic) of a mass of $10^{-3} \mathrm{~kg}$ experiences a force of $1.6 \times 10^{-4} \mathrm{~N}$ in a field of $\mathrm{B}_{\mathrm{z}}=1.8 \mathrm{~T}$ and a gradient of $\mathrm{dB} / \mathrm{dz}=17 \mathrm{~T} / \mathrm{m}$. A calcium sample (paramagnetic) of the same mass would be subject to a force of $-7.2 \times 10^{-4} \mathrm{~N}$. It is important that the material of the rotating ring is an insulator to avoid eddy currents. For the acceleration phase, the torus should contain hydrogen atoms. For transition into parallel space another material should be used. 
proton interaction is given by $\sigma=\frac{8 \pi}{3} r_{p}^{2}$,

where $r_{\mathrm{p}}$ is the classical proton radius, given by

$$
r_{p}=\frac{1}{4 \pi \epsilon_{0}} \frac{e^{2}}{m_{p} c^{2}}=w_{p h}^{2} \frac{\hbar}{m_{p} c} .
$$

For gravitophotons, $w_{\mathrm{ph}}$ has to be replaced by ${ }^{17}$ $N w_{\text {gp }}$, since in the conversion process from photon to gravitophotons , $N^{2}$ gravitophoton pairs are generated according to Eq. (35). The absorption cross section for a attractive (negative) gravitophoton particle by a material particle (here a proton or neutron is assumed) is given as

$$
\sigma_{g p}=\frac{8 \pi}{3}\left(N w_{g p a}\right)^{4}\left(\frac{\hbar}{m_{p} c}\right)^{2} .
$$

However, if the absorption is by an electron, the proton mass $m_{\mathrm{p}}$ has to be replaced by electron mass me. Therefore, the absorption cross section of a proton is larger by the factor $\left(m_{\mathrm{p}} / m_{\mathrm{e}}\right)^{2}$. Hence, the absorption of negative gravitophotons by electrons can be neglected.

For the generation (emission) of a gravitophoton pair from the vacuum by means of a virtual electron, the coupling constant is given by ${ }^{18}$ $w_{g p e}^{2}=G_{g p} \frac{m_{e}^{2}}{\hbar c}$. For the absorption of a negative gravitophoton by a proton, the coupling constant has the form $w_{g p a}^{2}=G_{g p} m_{p} \frac{m_{e}}{\hbar c}$. Using the absorption cross section for protons, the probability for this process is obtained as

$$
w=\frac{32}{3}\left(N w_{g p a}\right)^{4}\left(\frac{\hbar}{m_{p} c}\right)^{2} \frac{d}{d_{0}^{3}} Z .
$$

$d$ is the diameter of the torus, $d_{0}$ the diameter of the atom in its ground state, and $Z$ denotes the mass number of the atom. Since the first equation in Eqs. (35) describes the conversion of photons into $N^{2}$ gravitophoton pairs, $\alpha_{\mathrm{gp}}$ needs to be replaced by $N^{2} \alpha_{\text {gpp }}$. The force resulting from this conversion process, termed the Heim-Lorentz force [1], which is a gravitational force, has the form

$$
\boldsymbol{F}_{g p}=-w N^{2} \frac{\alpha_{g p}}{\alpha} e \mu_{0} \boldsymbol{v}^{T} \times \boldsymbol{H}
$$

The total force of the negative gravitophotons on the rotating body can be expressed in a form analogous to the Lorentz force

$$
\boldsymbol{F}_{g p}=-\Lambda_{p} e \mu_{0} \boldsymbol{v}^{T} \times \boldsymbol{H}
$$

where $\Lambda_{p}$ indicates that only proton and neutron absorption processes were considered. From Eqs. $(45,46) \quad \Lambda_{p}$ is determined as

$$
\frac{32}{3}\left(\frac{N w_{g p e}}{w_{p h}}\right)^{2}\left(N w_{g p a}\right)^{4}\left(\frac{\hbar}{m_{p} c}\right)^{2} \frac{d}{d_{0}^{3}} Z .
$$

$\Lambda_{p}$ (dimensionless) is a highly nonlinear function of the probability amplitude of the gravitophoton particle.

It is important to note that Eq. (47) only describes the acceleration stage of gravitophoton field propulsion. It should be noted that the current understanding is that the kinetic energy of the spacecraft is provided from the vacuum $^{19}$ and not from the magnetic field that is needed only to maintain the conversion process. The role of the magnetic field seems to be that of a catalyzer.

Conditions for entering a parallel space are given in Sec. (3.3). As will be seen, a completely different physical scenario needs to be established, requiring magnetic induction of some $30 \mathrm{~T}$ and torus material different from hydrogen.

\subsection{Technical Data for Acceleration Gravito- photon Field Propulsion}

Formulas $(47,48)$ will be used to calculate the strength of the gravitophoton field. To increase the strength of the interaction, a material containing hydrogen atoms should be used, because of the small value of $r$. For interstellar missions a different material should be used.

19 It is emphasized that the total energy extracted is 0 .
17 In the following, a distinction between emission (electron mass) and absorption (proton mass) of gravitophotons is necessary.

18 The value of $G_{g p}$ is given in Appendix $B$. 


\begin{tabular}{|r|r|r|r|}
\hline \multicolumn{1}{l|}{$\mathbf{n}$} & \multicolumn{1}{l|}{$\boldsymbol{N} w_{g p e}$} & \multicolumn{1}{c|}{$\begin{array}{c}\mu_{\mathbf{0}} \boldsymbol{H} \\
\mathbf{( T )}\end{array}$} & \multicolumn{1}{c|}{$\begin{array}{c}\text { Fgp } \\
\mathbf{( N )}\end{array}$} \\
\hline $10^{4}$ & $2.6 \times 10^{-14}$ & 2.0 & $7.14 \times 10^{-43}$ \\
\hline $10^{5}$ & $1.1 \times 10^{-5}$ & 6.3 & $3 \times 10^{1}$ \\
\hline $10^{6}$ & $1.5 \times 10^{-4}$ & 20.0 & $4.5 \times 10^{7}$ \\
\hline $10^{6}$ & $2.5 \times 10^{-4}$ & 50.0 & $1.45 \times 10^{9}$ \\
\hline
\end{tabular}

Table1: The right most column shows the total gravitophoton force in Newton that would act on the rotating ring. The force results from the absorption of the gravitophoton by a proton. The absorption through a proton results in a much larger force, so that in principle the interaction of a gravitophoton with an electron, regardless whether real or virtual, can be neglected. The number of turns of the magnetic coil is denoted by $n$, the magnetic induction is given in Tesla, and the current through the coil is $100 \mathrm{~A}$, except for the last row where 250 A were used. The mass of the rotating torus is 100 $\mathrm{kg}$, its thickness, $d$ (diameter) $0.05 \mathrm{~m}$, and its circumferential speed is $103 \mathrm{~m} / \mathrm{s}$. The wire cross section is $1 \mathrm{~mm} 2$. The meaning of the probability amplitude is given in the text.

For instance, if a larger spacecraft of $10^{5} \mathrm{~kg}$ with a rotating ring of $10^{3} \mathrm{~kg}$ needs to have a constant acceleration of $1 \mathrm{~g}$, a magnetic induc-

tion $\mu_{0} \boldsymbol{H}$ of some $13 \mathrm{~T}$ is needed together with a current density of $100 \mathrm{~A} / \mathrm{mm}^{2}$ and a coil of $4 \times 10^{5}$ turns for a value $N w_{g p e}=4.4 \times 10^{-5}$. The resulting force would be $10^{6} \mathrm{~N}$. Thus a launch of such a spacecraft from the surface of the earth seems to be technically feasible.

The high current in the superconducting coil produces a magnetic field $H$, that can be derived from a vector potential. Velocity $v_{\mathrm{k}}$ is the speed of the charge in the current loop or coil (Fig. 1). It is assumed that a superconductor is producing charge speeds of some $103 \mathrm{~m} / \mathrm{s}$. Together with the velocity $v_{k}^{T}$ of the rotating torus, this magnetic field generates the conversion potential according to Eq. (33). Photons are converted into negative and positive gravitophotons. Negative gravitophotons are absorbed by protons and neutrons, while positive gravitophotons do not interact, thus resulting in a measurable force.

\subsection{Space Flight in Parallel Space}

Gravitophoton propulsion takes place in two phases. In phase one a spacecraft is subject to acceleration in $\mathbb{R}^{4}$. Covering large interplanetary or interstellar distances, requires the transition into parallel space, which is phase two of the field propulsion system.

A complete mathematical discussion of parallel space cannot be given in the framework of this paper. Therefore, only the salient physical features and their consequences are presented.

As was shown in Chap. 2, an electromagnetic field can be transformed into a gravitational like field, producing both negative and positive gravitophotons from the vacuum. The fundamental fact for transition into parallel space is that the gravitational potential of a spacecraft with mass $M$ is reduced by the interaction of the positive gravitophotons with gravitons and their conversion into (repulsive) vacuum particles. There is an additional conversion equation, similar to Eqs. (35), for converting the gravitons of the spacecraft together with the positive gravitophotons into the postulated vacuum particles (or quintessence particles), thus reducing the gravitational potential of the spacecraft. The gravitational potential, $\Phi$, is given by

$$
\Phi(\boldsymbol{r})=-\int \frac{G \rho_{g}\left(\boldsymbol{r}^{\prime}\right)}{\left|\boldsymbol{r}-\boldsymbol{r}^{\prime}\right|} d^{3} r^{\prime}
$$

which implies that $\Phi$ obeys the Poisson equation

$$
4 \pi G M=\oint \nabla \Phi\left(\boldsymbol{r}^{\prime}\right) \cdot d \boldsymbol{S}
$$

where integration is over the closed surface $S$ of a volume $V$ in physical space that contains the spacecraft. Following the arguments by Penrose [5, 18] (violation of causality) and by Krauss [3] (a signal is needed to tell spacetime to warp, but its speed itself cannot exceed $c$ ), $G R$ clearly does not allow to travel faster than the speed of light in spacetime $\mathbb{R}^{4}$. Since $a b-$ sorption of positive gravitophotons is reducing $\Phi$, this would require either the mass of the spacecraft to be reduced in $\mathbb{R}^{4}$, or the gravitational constant $G$ to become smaller, owing to Eq. (49). As a consequence of a reduced mass, conservation of momentum would require a 
velocity $c^{\prime}>c^{20}$ in $\mathbb{R}^{4}$, which has to be ruled out. The decision for a reduced gravitational constant $G^{\prime}<G$ in $\mathbb{R}^{4}$ is more difficult. To this end, we refer to quantum gravity theory [26], according to which area is quantized, that is

$$
\tau=16 \pi \frac{\hbar G}{c^{3}} \sqrt{j(j+1)} .
$$

The minimal surface $\tau=8 \sqrt{3} \pi \frac{\hbar G}{c^{3}}$ is obtained for $j=1 / 2$. Therefore any physical phenomenon that requires a gravitational constant $G^{\prime}<G$ or a speed of light $c^{\prime}>c$ in $\mathbb{R}^{4}$ has to be ruled out, violating the fact that $\mathrm{T}$ is the minimum surface. On the other hand, because of positive gravitophoton action, $\Phi$ actually is reduced, and thus the concept of parallel space (or parallel universe or multiverse) is introduced, denoted as $\mathbb{R}^{4}(n)$ with $n \in \mathbb{N}$. For $n=1$, $v(1):=v$ (velocity of the spacecraft) and $\mathbb{R}^{4}(1):=$ $\mathbb{R}^{4}$. It is postulated that a spacecraft, under certain conditions, stated below by Eq.(52), will be able to transition into such a parallel space. For $G(n)=G / n, M(n)=n M$, and $c(n)=n c$, the spacecraft would transition into $n^{\text {th }}$-parallel space $\mathbb{R}^{4}(n)$. An indirect proof for the existence of parallel spaces could be the observed phenomenon of dark matter, see Sec. (4.4).

A parallel space $\mathbb{R}^{4}(n)$, in which covariant physical laws with respect to $\mathbb{R}^{4}$ exist, is characterized by the scaling transformation

$$
\begin{aligned}
& x_{i}(n)=\frac{1}{n^{2}} x(1), i=1,2,3 ; t(n)=\frac{1}{n^{3}} t(1) \\
& v(n)=n v(1) ; c(n)=n c(1) \\
& G(n)=\frac{1}{n} G ; \hbar(n)=\hbar ; n \in \mathbb{N} .
\end{aligned}
$$

The fact that $n$ must be an integer stems from the requirement in $L Q T$ for a smallest length scale. Hence only discrete and no continuous transformations are possible. The Lorentz transformation is invariant with regard to the transformations of Eqs. (51) ${ }^{21}$. In other words, physical laws should be covariant under dis-

20 This is in contrast to [2] where a reduction in mass was assumed, and a velocity $c^{\prime}>c$ was postulated. The important fact, however, is the reduction of the gravitational potential.

21 It is straightforward to show that Einstein's field equations as well as the Friedmann equations are also invariant under dilatations. crete (quantized) spacetime dilatations (contractions).

The two important questions to be addressed, concern the value $n$, in particular, how it is influenced by experimental parameters, and the back-transformation from $\mathbb{R}^{4}(n) \rightarrow \mathbb{R}^{4}$. The result of the back-transformation must not depend on the choice of the origin of the coordinate system in $\mathbb{R}^{4}$. For the lack of space, the detailed discussion for the two mappings from $\mathbb{R}^{4} \rightarrow \mathbb{R}^{4}(n) \rightarrow \mathbb{R}^{4}$ cannot be presented, but the result is that the spacecraft has moved a distance $n v \Delta T$ when reentering $\mathbb{R}^{4}$. This mapping for the transformation of distance, time and velocity differences is not the identity matrix that is, the second transformation is not the inverse of the first transformation ${ }^{22}$. The spacecraft is assumed to be leaving $\mathbb{R}^{4}$ with velocity $v$, and $\Delta T$ denotes the time difference between leaving and reentering $\mathbb{R} 4$, as measured by an observer in $\mathbb{R}^{4}$. It should be noted that energy conservation in $\mathbb{R}^{4}$ has to be satisfied that is, the energy of the spacecraft remains unchanged upon reentering (provided no acceleration occurred in $\mathbb{R}^{4}(n)$ ), given by the relativistic formula

$$
M(v) c^{2}=\frac{M_{0} c^{2}}{\sqrt{1-\left(\frac{v(1)}{c(1)}\right)^{2}}} \text { and } \frac{v(1)}{c(1)}=\frac{v(n)}{c(n)}=\frac{n v(1)}{n c(1)} .
$$

The value of $n$ is obtained from the following formula, Eq. (52), relating the field strength of the gravitophoton field, $g_{g p}^{+}$, with the gravitational field strength, $g_{g}$, produced by the spacecraft itself,

$$
n=\frac{g_{g p}^{+}}{g_{g}} \frac{G_{g p}}{G} .
$$

This formula will be used in the next section to calculate the conditions for a transition into parallel space. The positive gravitophoton field is generated together with the negative gravitophoton field, and, because of energy conserva-

22 In other words, a quantity $v(\mathrm{n})=n v(1)$, obtained from a quantity of $\mathbb{R}^{4}$, is not transformed again when going back from $\mathbb{R}^{4}(\mathrm{n})$ to $\mathbb{R}^{4}$. This is in contrast to a quantity like $\Delta T(n)$ that transforms into $\Delta T$. The reason for this unsymmetrical behavior is that $\Delta T(n)$ is a quantity from $\mathbb{R}^{4}(\mathrm{n})$ and thus is being transformed. 
tion, has the same value. Therefore, its strength can be directly calculated from Eq. (47). Assuming a magnetic induction of $30 \mathrm{~T}$, a current density of $230 \mathrm{~A} / \mathrm{mm}^{2}$, and $4 \times 10^{5}$ turns for the magnetic coil, the positive gravitophoton field should result in an acceleration of $3 \times 10^{2} \mathrm{~m} / \mathrm{s}^{2}$, in direct vicinity of the torus. Some $10 \mathrm{~m}$ away from the torus the acceleration should be some $0.1 \mathrm{~g}$ or $1 \mathrm{~m} / \mathrm{s}^{2}$. This value for $g_{g p}^{+}$is being used in calculating the value of $n$ for the interplanetary and interstellar missions of Sec. (3.4). The rotating torus generates pairs of both negative and positive gravitophotons. Negative gravitophotons are absorbed by protons and neutrons, while the remaining positive gravitophotons interact with the gravitons of the spacecraft, being converted into vacuum particles, thus reducing the gravitational potential of the spacecraft. Eq. (52) then determines the condition for transition into parallel space $\mathbb{R}^{4}(n)$. Since $n$ is an integer, the effect is quantized and requires a threshold value for $g_{g p}^{+}$.

\subsection{Lunar, Interplanetary, and Interstellar Missions}

In the following we discuss three missions, a lunar mission, a Mars mission, and an interstellar mission. From the numbers provided, it is clear that gravitophoton field propulsion, if feasible, cannot be compared with chemical propulsion or any other currently conceived propulsion system. Furthermore, an acceleration of $1 \mathrm{~g}$ can be sustained during flight for lunar missions.

Gravitophoton field propulsion is a two-stage process. First, an acceleration is achieved by the absorption of negative gravitophotons through the protons and neutrons of the torus material. In the second stage, a transition into a parallel space takes place that leads to a huge increase by a factor $n$, see Eq. (52), in speed with regard to our spacetime $\mathbb{R}^{4}$ (see previous section).

For lunar missions only stage one is needed. The high values of magnetic induction for a transition to parallel space are not needed. For the lunar mission a launch from the surface of the earth is foreseen with a spacecraft of a mass of some $1.5 \times 10^{5} \mathrm{~kg}(150 \mathrm{t})$. With a magnetic induction of $20 \mathrm{~T}$, compare Table (1), a rotational speed of the torus of $v^{\mathrm{T}}=10^{3} \mathrm{~m} / \mathrm{s}$, and a torus mass of $2 \times 10^{3} \mathrm{~kg}$, an acceleration larger than $1 \mathrm{~g}$ is produced so that a launch is possible. Assuming an acceleration of $1 \mathrm{~g}$ during flight, the first half of the distance, $d_{\mathrm{M}}$, to the moon is covered in some 2 hours, which directly follows from $t=\sqrt{\frac{2 d_{M}}{g}}$, resulting in a total flight time of 4 hours. Since the distance is very short, entering parallel space is not necessary.

A Mars mission, under the same assumptions as a flight to the moon, that is, if only stage one of the field propulsion is used, would need an acceleration phase of 414 hours. The final velocity would be $v=\mathrm{gt}=1.49 \times 106 \mathrm{~m} / \mathrm{s}$. This would be a hypothetical value only, if the amount of energy needed would have to be provided by the electromagnetic field. However, this kinetic energy is extracted from the vacuum, although the total energy extracted from the vacuum that is in the form of negative and positive gravitophotons, is zero. The total flight time to Mars with acceleration and deceleration is 34 days.

Using stage two field propulsion that is entering parallel space, a transition is possible at a speed of some $3 \times 10^{4} \mathrm{~m} / \mathrm{s}$ that will be reached after approximately 1 hour at a constant acceleration of $1 \mathrm{~g}$. The transition into parallel space has the effect that the velocity increases to $0.4 c$. In that case, total flight time would be reduced to some 2.5 hours $^{23}$.

For an interstellar mission, the concept of parallel space is indispensable. An acceleration phase of some 34 days with $1 \mathrm{~g}$ would result in a final velocity of one per cent of the speed of light, $0.01 c$. Again, gravitophoton field propulsion would obtain the kinetic energy from the vacuum. The transition into parallel space would need a repulsive strength of the gravitophoton field (positive gravitophotons), producing an acceleration $g_{g p}^{+}=1 \mathrm{~m} / \mathrm{s}^{2}$ at some 10 $\mathrm{m}$ (order of magnitude) away from the space-

23 Today's propulsion systems demand long mission times to Mars, and adequate protection must be provided against radiation hazards. Reinforced polyethylene (hydrogen content) is being investigated, but may significantly increase the mass of the spacecraft. 
craft. The gravitational field strength of the spacecraft itself with mass $10^{5} \mathrm{~kg}$ is given by

$$
g_{g}=G \frac{M}{R^{2}} \approx 6.67 \times 10^{-8} \mathrm{~m} / \mathrm{s}^{2} . \quad \text { Inserting these }
$$

values into Eq. (52), transition into parallel space would cause a velocity gain by a factor of $n=3.3 \times 10^{4}$, resulting in an effective speed of $3.3 \times 10^{2} c$. This means for an observer in $\mathbb{R}^{4}$ that the spacecraft seems to have moved at such a superluminal speed. A distance of 10 light-years could be covered within 11 days. The deceleration phase requires another 34 days, so that a one-way trip will take about 80 days to reach, for instance, the star Procyon that is $3.5 \mathrm{pc}^{24}$ from earth. There are about 30 known stars within a radius of 13 light-years from earth.

\section{Cosmology from HQT and LQT}

Despite the successes of modern physics, the most fundamental questions, as will be shown in the subsequent section, cannot be answered. It is clear that the current status of physics is far from being the final theory. Therefore, to argue that something is not possible based on the insights of current theory, is not necessarily true. Advanced propulsion systems do require novel physics beyond present concepts. Quantum gravity could be a key theory.

\subsection{Deficiencies in Current Fundamental Physical Theories}

The wave picture of $Q T$ does not describe the particle aspect occurring in Nature. The current standard model, trying to describe particle features by introducing new additional quantum numbers, has not been successful in explaining fundamental physics such as the measured mass spectrum of elementary particles and their lifetimes [17], neither can the very nature of matter be explained. It is also not known how many fundamental physical interactions exist, neither is the dimensionality of space, nor can quantum numbers be derived. If all the quantum numbers describing an elementary particle have to be introduced ad hoc, it would be difficult to imagine such a particle as elementary. According to Heim this is actually not the case [4].

24 Parsec is a distance and $1 \mathrm{pc}=3.26 \mathrm{ly}$.
Despite its success in predicting the mass of some new particles, quantum electrodynamics and quantum chromodynamics are plagued by logical inconsistencies, i.e., by infinities. Renormalization gets rid of these infinities by subtracting infinities from infinities in order to get something finite. This is only justified by the meaningful results that follow from this physically inconsistent process [17].

A major question therefore is on the roles of and the relationship between $G R$ and $Q T$ with regard to the explanation of physical reality, and how these theories could be modified to describe the material world in a consistent way. Furthermore, it has to be clarified whether current theory has found all possible physical interactions. For instance, many scientists have already guessed that an interaction between electromagnetism and gravitation should exist [3], not contained in the laws of current physics.

Einstein's $G R$ of 1915 is a revolutionary theory that could provide the framework of a geometrization of all physical interactions: gravity is no longer treated as a force, but is represented by the curvature of spacetime. Over the last two decades $G R$ has been tested to be correct to one part in $10^{14}$ by measuring the shrinking in the orbit of the Hulse-Taylor binary pulsar (two neutron stars where one is a pulsar) [23], i.e., measuring the periodic Doppler shift of the pulsar's radiation. The energy loss is attributed to gravitational waves. Hence, the accuracy of GR is greater than for $Q T$ or even $Q E D$. If gravity is not to be treated different from all other physical interactions, and since it is confirmed to such a marvelous accuracy, it seems to be justified to use this approach for all physical forces, and also to apply this concept to the subatomic range. Therefore, in Heim's approach Einstein's theory serves as the paradigm, on which all other physical theories are to be modeled.

Consequently, Heim has extended four dimensional spacetime to higher dimensions (additional imaginary coordinates), constructing a poly-metric, and assigning all physical interactions their proper metric. In the subatomic range, the quantization of spacetime proved to be necessary. In this way a unified theory was obtained. In other words, physics is geometry, and matter is geometry, too. 
When Heim solved the resulting eigenvalue equations, the mass spectrum of ponderable particles was obtained as described in [6, 12], along with their quantum numbers. Elementary particles themselves are dynamical, cyclic structures built from metrons, elemental surfaces with spin, in a hierarchical way [4, 7]. Thus, a complete geometrization of Nature is achieved, reducing matter to a cyclic feature of higher-dimensional space itself. Most important, however, if the admissible metric combinations are investigated, they lead to the conclusion that six fundamental interactions must exist.

\subsection{Common Concepts in HQT and LQT}

Though HQT is based on geometrical aspects in a 6,8 , or 12 -dimensional space, it is neither continuous nor smooth, but contains an elemental surface area, the metron, equipped with a spin vector. A quantized volume element, bounded by metron surfaces, may have all spins pointed outward (exogen) or all spins directed inward (endogen). Because of the isotropy of space the metronic lattice (also called $\tau$-lattice) contains both types of volumes. Empty space comprises a dynamic lattice of orthogonal elemental cells. Any lattice that deviates from this Cartesian lattice is called a $h y$ perstructure and is capable of describing physical events. The curvature of space, inherent to a hyperstructure, is termed condensation, since the number of metrons (because of their fixed size) on a curved surface must be larger than on its projection into Euclidean space. Hyperstructures are described by an eigenvalue problem, leading to quantized levels of space that are associated with real physical states. Space itself is ascribed a structural potential, meaning that fundamental ontological qualities of space itself appear as geometric structures.

When compared to recent ideas from loop quantum gravity, there is similarity on the ideas of the quantized structure of spacetime. Furthermore, both theories start from Einstein's $G R$, requiring background independence (no fixed coordinate system, but a dynamical evolving geometry) and independence on the coordinate values (the physics must not depend on the choice of coordinate system, also termed diffeomorphism invariance). Spin networks in quantum loop theory [11] and
Heim's hyperstuctures are both used to represent dynamical quantum states of space. Heim uses a higher-dimensional space, for instance in 6D space there are 30 metron surfaces bounding a volume, to construct a poly-metric to unify all physical forces [4]. Loop quantum theory currently is formulated in $4 \mathrm{D}$ spacetime and does not explicitly rely on a metric. As mentioned by its authors the extension to higher dimensional spaces should be possible. However, if physical quantities need to be computed, a metric eventually is indispensable ${ }^{25}$.

While Heim's derivation of the metron is based on heuristic physical arguments, the picture of quantum spacetime in $L Q T$ is on firm mathematical ground, see, for instance, [28]. It seems that Heim's approach resembles the Bohr model of the atom, while $L Q T$ is more like the Heisenberg picture. On the other hand, Heim constructed a unified theory through the introduction of a poly-metric in a higher-dimensional space, predicting the existence of two additional fundamental interactions. Moreover, he constructed a set of eigenvalue equations from the field equations of $G R$ resulting in the derivation of the mass spectrum for elementary particles $[4,6,12]$. Nothing can be said at present whether $H Q T$, in analogy to Einstein's $G R$, could be cast into the framework of Ashtekar's novel formulation in which $G R$ is in a similar form to Yang-Mills theory $[24,26]^{26}$. It should be noted that according to Heim any material particle has its associated proper hermetry form, and, as a consequence of this, there is a unity of field and field source.

One important consequence of any theory based on quantized surfaces and volumes is that the picture of an elementary particle as a point-like entity [17] becomes untenable. According to $G R$ and $Q T$, point-like particles with mass will collapse into black holes [26] and disappear, which is in stark contrast to the very existence of elementary particles. With the classical radius of the electron of some

25 This includes the fact that all intermediate volumes connecting initial and final volumes on the lattice or the spin network could be identified and numbered. To this end, Heim developed his own mathematics and coined the term selector, see Chap. 3 in [6].

26 The idea for the comparison of the two theories is due to Dr. Jean-Luc Cambier, Senior Scientist, Propulsion Directorate, EAFB. 
$3 \times 10^{-15} \mathrm{~m}$, and a metron size of some $10^{-70} \mathrm{~m}^{2}$, about $10^{41}$ metrons are needed to cover the surface of the electron. An electron therefore must be a highly complex geometrical quantity. According to Heim, elementary particles having rest mass constitute self-couplings of free energy. They are indeed elementary as far as their property of having rest mass is concerned, but internally they possess a very subtle, dynamic structure. For this reason they are elementary only in a relative sense. The argument, put forward by C. Rovelli [25] that hadrons cannot be elementary, because there are too many quantum numbers needed for their description is not necessarily true. Heim, in his 1977 paper [4], uses a set of 12 quantum numbers to describe an elementary particle, and claims that these quantum numbers can be reduced to a single quantum number $k=1$ or $k=2$ and the decision $\epsilon= \pm 1$ for particle or antiparticle.

\subsection{Cosmological Consequences}

Going back in time, the volume of the universe becomes smaller and smaller [1, 2, 28]. Because of the quantization of area and volume a singularity in space cannot develop. The universe would have started with the smallest possible volume, namely a volume being proportional to the Planck length cubed, $\ell_{p}^{3}$. According to Heim this was, however, not the case, since the metron size, T, is increasing when going back in time while, in parallel, the number of metrons decreases, until there is a single metron only, covering the primeval universe.

This links the dynamical evolution with the initial conditions, and allows for one single choice only. Thus, the problem of initial conditions for the universe is solved by quantization ${ }^{27}$.

Moreover, in HFT, gravitation is attractive between a distance $R_{-}<r<\rho$, and becomes slightly repulsive for $\rho<r<R_{+}$and goes to 0 for $r>R_{+}$. $R_{\text {. }}$ is a lower bound for gravitational

27 No discussion is intended of pre-structures before the universe came into being via creation of the first metron, see [9]. The idea presented there, the apeiron, is similar to Penrose's ideal mathematical world [5]. structures, comparable to the Schwarzschild radius. The distance at which gravitation changes sign, $\rho$, is some 46 Mparsec. $R_{+}$denotes an upper bound and is some type of Hubble radius, but is not the radius of the universe, instead it is the radius of the optically observable universe. Gravitation is zero beyond the two bounds, that is, particles smaller than $R$. cannot generate gravitational interactions.

Consequently, the cosmological redshift is explained by the repulsive gravitational potential, and not by the Doppler shift. A more detailed discussion is given in Sec. 5 of [2]. According to Heim the age of the universe is some $10^{127}$ years. Matter, as we know it, was generated only some 15 billion years ago, when T, the metron size, became small enough.

A very interesting fact is that in $H Q T$ the constants $G, \hbar, \epsilon_{0}, \mu_{0}$, and $\mathrm{T}$ are all functions of the diameter, $D$, of the primeval universe in which our optical universe is embedded. Since matter is very recent in comparison with the age of the primeval universe, these constants remained practically unchanged for the last 15 billion years (for more details see Sec. 2.2 [1]).

Although HFT and LQT [28] provide a different cosmogony, they both solve the singularity problem based on quantized spacetime as well as the problem of initial conditions. Both theories make proposals that can be confronted with cosmological observations.

\subsection{Dark Matter}

The existence of parallel spaces could indirectly support the observed amount of dark matter. According to our computations, the mass in all parallel spaces $\mathbb{R}^{4}(n)$ should be some 7 times the mass in $\mathbb{R}^{4}$, and should be felt via gravitational interaction in $\mathbb{R}^{4}$. Dark matter therefore should be around $28 \%$ with regard to the sum of visible and non-visible matter (currently at about 4\%) in $\mathbb{R}^{4}$.

\subsection{Dark Energy}

Dark energy is explained by hermetry form $H_{10}$, and is the sixth fundamental interaction proposed by $H Q T$. This interaction is termed vacuum field and is repulsive. 


\section{Conclusions and Future Work}

The authors are aware of the fact that the current paper contains shortcomings with regard to mathematical rigor, and also proposes two highly speculative concepts ${ }^{28}$. It should be kept in mind, however, that any type of field propulsion ${ }^{29}$ necessarily must exceed conventional physical concepts. In addition, the important conversion equations for photons into gravitophotons and gravitophotons into vacuum (quintessence) particles were not derived, simply for the lack of space, see [9].

The first of these concepts is the complete geometrization of physics, extending the Einsteinian picture to all physical interactions. This requires an 8D space, termed Heim space, comprising four subspaces that are used to construct a polymetric. Each of the partial metric, termed hermetry form (see glossary), represents a physical interaction or interaction particle. As a consequence, there are six fundamental interactions, instead of the four known ones. The two additional interactions are gravitational like, one allowing the conversion of photons into hypothetical gravitophoton particles, generated from the vacuum that come in two forms, namely repulsive and attractive. This interaction is the basis of the proposed gravitophoton field propulsion. Whether or not the mechanism, described in detail in Chap. 2, on which this field propulsion is based, is true can only be decided by experiment. Consequently, an experimental set up along with calculated gravitophoton forces was presented.

The sixth interaction is identified with the quintessence and is repulsive, thus giving an explanation for the observed expansion of the universe.

The second concept concerns the transition of a material object into a so called parallel space (i.e., there are other universes), in which the limiting speed is $n c{ }^{30}$, where $c$ is vacuum speed of light and $n>1$ is an integer (accord-

28 The reader should remember the remark by A.Clarke that any future technology is indistinguishable from magic.

29 Field propulsion means that the propulsion mechanism is not based on the classical principle of momentum conservation as being used in the rocket equation.

30 The problem of causality is still present. Also, the qestion of navigation a spacecraft in ing to our computations there is an upper limit of $6.6 \times 10^{10}$ for $n$ ). The concept of parallel spaces could indirectly be justified, since it also can be used in calculating the amount of dark matter. Additional matter exists in these parallel spaces, and via gravitational interaction may cause the observed effects in our universe, attributed to dark matter. The question of navigation in a parallel space with regard to $\mathbb{R}^{4}$ cannot be answered at present.

Substantial work needs to be done to refine the calculations for the gravitophoton force and the experimental setup.

With respect to the theoretical framework of $H Q T$, the authors feel that $H Q T$ could benefit much from the mathematical structure of $L Q T$. Recent $L Q T$ seems to have the potential to revolutionize the physical picture of spacetime. Most interesting, from the concept of minimal surface it follows directly that superluminal speeds or the reduction of $G$ are prohibited in our spacetime. $H Q T$ postulating the existence of gravitophotons that can reduce a gravitational potential, thus requires the concept of parallel space.

It is interesting to see that $H Q T$, being developed much earlier than $L Q T$, is based on similar assumptions. Therefore, in view of the progress in quantum gravity and the similarities between the two theories, the attempt to cast Heim's theory into the modern formulation of loop theory seems to be worthwhile, because, if such a formulation can be achieved, it would be a clear hint that these additional interactions actually might exist, having an enormous impact on the technology of future flight and transportation in general.

\section{Acknowledgment}

The authors dedicate this paper to Prof. P. Dr. Dr. A. Resch, director of IGW at Innsbruck University, at the occasion of his $70^{\text {th }}$ birthday. Prof. Resch has not only published the theories of Burkhard Heim, but was also instrumental in editing the complete scientific work of Heim. This proved to be an enormously difficult and time-consuming task, since Heim as an author, because of his handicap, was not able to proofread complex manuscripts, and thus could not help with the typesetting of the complex formulas. The authors also wish to acknowledge the voluminous scientific work 
of Dr. Resch, Imago Mundi, whose prime subject was and is the creation of a consistent Weltbild, acceptable both in science and humanities to bridge the gap that currently divides these two disciplines.

The authors are very much indebted to Dipl.Phys. I. von Ludwiger, currently secretary of the Heim Forschungskreis and former manager and physicist at DASA, for making available relevant literature and for publishing a recent article about Heim's mass formula calculating the mass spectrum of elementary particles, providing a comparison with latest experimental results.

The second author was partly funded by Arbeitsgruppe Innovative Projekte (AGIP), Ministry of Science and Education, Hanover, Germany.

We are grateful to Prof. Dr. T. Waldeer of the University of Applied Sciences at Salzgitter Campus for helpful discussions. The help of $T$. Gollnick and O. Rybatzki of the University of Applied Sciences at Salzgitter Campus in the preparation of this manuscript is appreciated.

\section{Appendix A: Mass Spectrum of Ele- mentary Particles}

Mass spectrum of elementary particles as calculated from HQT together with comparisons of recent experimental data are available from

http://www.uibk.ac.at/c/cb/cb26/heim/index.ht $\mathrm{ml}$.

\section{Appendix B: Gravitational Coupling Constants for the three gravitational interactions as obtained from number theory (see [9]).}

For the table below, it should be noted that all coupling constants are computed with respect to the proton mass. In this regard, wq is a fictitious value only, since there is no emitting real proton mass. Most likely, the generation energy for vacuum particles is the Planck mass. Dimensional units used for table entries are kg, $\mathrm{m}$, and $\mathrm{s}$.

\begin{tabular}{|l|l|l|}
\hline \multicolumn{3}{|c|}{ Gravitational Coupling Constants } \\
\hline wg & $7.683943001 \times 10^{-20}$ & graviton \\
\hline wgp & $1.14754864 \times 10^{-21}$ & gravitophoton \\
\hline wq & $1.603810891 \times 10^{-28}$ & $\begin{array}{l}\text { vacuum particle } \\
\text { (quintessence) }\end{array}$ \\
\hline Ggp & $1 / 67^{2} \mathrm{G}$ & $\left(\frac{w_{g}}{w_{g p}}\right)^{2}=\frac{G}{G_{g p}}$ \\
\hline Gq & $4.3565 \times 10^{-18} \mathrm{G}$ & $\left(\frac{w_{g}}{w_{q}}\right)^{2}=\frac{G}{G_{q}}$ \\
\hline$\alpha g$ & $5.904298005 \times 10^{-39}$ & $\alpha_{g}=\frac{G m_{p}^{2}}{\hbar c}$ calculated \\
\hline $\mathrm{G}$ & $6.6722037 \times 10^{-11}$ & calculated \\
\hline $\mathrm{Ge}$ & $6.673(10) \times 10^{-11}$ & experimental value \\
\hline
\end{tabular}

\section{Glossary ${ }^{31}$}

aeon Denoting an indefinitely long period of time. The aeonic dimension, $x_{6}$, can be interpreted as a steering structure governed by the entelechial dimension toward a dynamically stable state.

anti-hermetry Coordinates are called anti-hermetric if they do not deviate from Cartesian coordinates, i.e., in a space with antihermetric coordinates no physical events can take place.

condensation For matter to exist, as we are used to conceive it, a distortion from Euclidean metric or condensation, a term introduced by Heim, is a necessary but not a sufficient condition.

conversion amplitude Allowing the transmutation of photons into gravitophotons, $w_{\text {ph_gp }}$ (electromagnetic-gravitational interaction), and the conversion of gravitophotons into quintessence particles, $w_{\text {gp_ } \mathrm{q}}$ (gravitational-gravitational interaction).

coupling constant Value for creation and destruction of messenger (virtual) particles, relative to the strong force (whose value is

31 A more comprehensive glossary is available under www.cle.de/hpcc, see Publications 
set to 1 in relation to the other coupling constants).

coupling potential between photon-gravitophoton (Kopplungspotential) As coupling potential the first term of the metric in Eq. (23) is denoted, that is $g_{i k}^{(g p)}$. The reason for using the superscript $\mathrm{gp}$ is that this part of the photon metric equals the metric for the gravitophoton particle and that a $\rightarrow$ sieve (conversion) operator exists, which can transform a photon into a gravitophoton by making the second term in the metric anti-hermetric. In other words, the electromagnetic force can be transformed into a repulsive gravitational like force, and thus can be used to accelerate a material body.

cosmogony (Kosmogonie) The creation or origin of the world or universe, a theory of the origin of the universe (derived from the two Greek words kosmos (harmonious universe) and gonos (offspring)).

entelechy (Greek entelécheia, objective, completion) used by Aristotle in his work The Physics. Aristotle assumed that each phenomenon in nature contained an intrinsic objective, governing the actualization of a form-giving cause. The entelechial dimension, $x_{5}$, can be interpreted as a measure of the quality of time varying organizational structures (inverse to entropy, e.g., plant growth) while the aeonic dimension is steering these structures toward a dynamically stable state. Any coordinates outside spacetime can be considered as steering coordinates.

geodesic zero-line process This is a process where the square of the length element in a 6- or 8-dimensional Heim space is zero.

gravitational limit(s) There are three distances at which the gravitational force is zero. First, at any distance smaller than R, the gravitational force is 0 . Second,

gravitophoton (field) Denotes a gravitational like field, represented by the metric subtensor, $g_{i k}^{(g p)}$, generated by a neutral mass with a smaller coupling constant than the one for gravitons, but allowing for the possibility that photons are transformed into gravitophotons. Gravitophoton particles can be both attractive and repulsive and are always generated in pairs from the vacuum under the presence of virtual electrons. The total enery extracted from the vacuum is zero, but only attractive gravitophotons are absorbed by protons or neutrons. The gravitophoton field represents the fifth fundamental interaction. The gravitophoton field generated by repulsive gravitophotons, together with the $\rightarrow v a c$ uum particle, can be used to reduce the gravitational potential around a spacecraft.

graviton (Graviton) The virtual particle responsible for gravitational interaction.

Heim-Lorentz force Resulting from the newly predicted gravitophoton particle that is a consequence of the Heim space $\mathbb{R}^{8}$. A metric subtensor is constructed in the subspace of coordinates $\mathrm{I}^{2}, \mathrm{~S}^{2}$ and $\mathrm{T}^{1}$, denoted as hermetry form $\mathrm{H}_{5}$, see $[1,6,7]$. The equation describing the Heim-Lorentz force has a form similar to the electromagnetic Lorentz force, except, that it exercises a force on a moving body of mass $m$, while the Lorentz force acts upon moving charged particles only. In other words, there seems to exist a direct coupling between matter and electromagnetism. In that respect, matter can be considered playing the role of charge in the Heim-Lorentz equation. The force is given by $\boldsymbol{F}_{g p}=\Lambda_{p} q \mu_{0} \boldsymbol{v}^{T} \times \boldsymbol{H}$. Here $\Lambda_{p}$ is a coefficient, $v^{\mathrm{T}}$ the velocity of a rotating body (e.g., torus, insulator) of mass $\mathrm{m}$, and $H$ is the magnetic field strength. It should be noted that the gravitophoton force is 0 , if velocity and magnetic field strength are perpendicular. Thus, any experiment that places a rotating disk in a uniform magnetic field that is oriented parallel or antiparallel to the axis of rotation of this disk, will measure a null effect.

hermetry form (Hermetrieform) The word hermetry is an abbreviation of hermeneutics, in our case the semantic interpretation of the metric. To explain the concept of a hermetry form, the space $\mathbb{R}^{6}$ is considered. 
There are 3 coordinate groups in this space, namely $s_{3}=\left(\xi_{1}, \xi_{2}, \xi_{3}\right)$ forming the physical space $\mathbb{R}^{3}, s_{2}=\left(\xi_{4}\right)$ for space $\mathrm{T}^{1}$, and $s_{1}=\left(\xi_{5}, \xi_{6}\right)$ for space $\mathrm{S}^{2}$. The set of all possible coordinate groups is denoted by $S=\left\{s_{1}, s_{2}, s_{3}\right\}$. These 3 groups may be combined, but, as a general rule (stated here without proof, derived, however, by Heim from conservation laws in $\mathbb{R}^{6}$, (see p. 193 in [6])), coordinates $\xi_{5}$ and $\xi_{6}$ must always be curvilinear, and must be present in all metric combinations. An allowable combination of coordinate groups is termed hermetry form, responsible for a physical field or interaction particle, and denoted by $H$. $H$ is sometimes annotated with an index such as $H_{10}$, or sometimes written in the form $H=\left(\xi_{1}, \xi_{2}, \ldots\right)$ where $\xi_{1}$, $\xi_{2}, \ldots \in \mathrm{S}$. This is a symbolic notation only, and should not be confused with the notation of an n-tuple. From the above it is clear that only 4 hermetry forms are possible in $\mathbb{R}^{6}$. It needs a Heim space $\mathbb{R}^{8}$ to incorporate all known physical interactions. Hermetry means that only those coordinates occurring in the hermetry form are curvilinear, all other coordinates remain Cartesian. In other words, $H$ denotes the subspace in which physical events can take place, since these coordinates are non-euclidean. This concept is at the heart of Heim's geometrization of all physical interactions, and serves as the correspondence principle between geometry and physics.

hermeneutics (Hermeneutik) The study of the methodological principles of interpreting the metric tensor and the eigenvalue vector of the subspaces. This semantic interpretation of geometrical structure is called hermeneutics (from the Greek word to interpret).

homogeneous The universe is everywhere uniform and isotropic or, in other words, is of uniform structure or composition throughout.

hyperstructure (Hyperstruktur) Any lattice of a Heim space that deviates from the isotropic Cartesian lattice, indicating an empty world, and thus allows for physical events to happen.

isotropic The universe is the same in all directions, for instance, as velocity of light transmission is concerned measuring the same values along axes in all directions.

partial structure (Partialstruktur) For instance, in $\mathbb{R}^{6}$, the metric tensor that is hermitian comprises three non-hermitian metrics from subspaces of $\mathbb{R}^{6}$. These metrics from subspaces are termed partial structure.

poly-metric The term poly-metric is used with respect to the composite nature of the metric tensor in $8 \mathrm{D}$ Heim space. In addition, there is the twofold mapping $\mathbb{R}^{4} \rightarrow \mathbb{R}^{8} \rightarrow \mathbb{R}^{4}$. It can be shown that when this mapping is applied to the Christoffel symbols they take on tensor character.

probability amplitude With respect to the six fundamental interactions predicted from the $\rightarrow$ poly-metric of the Heim space $\mathbb{R}^{8}$, there exist six (running) coupling constants. In the particle picture, the first three describe gravitational interactions, namely $w_{\mathrm{g}}$ (graviton, attractive), $w_{\mathrm{gp}}$ (gravitophoton, attractive and repulsive), $w_{\mathrm{q}}$ (quintessence, repulsive). The other three describe the well known interactions, namely $w_{\text {ph }}$ (photons), $w_{\mathrm{w}}$ (vector bosons, weak interaction), and $w_{\mathrm{s}}$ (gluons, strong interaction). In addition, there are two $\rightarrow$ conversion amplitudes predicted that allow the transmutation of photons into gravitophotons (electromagnetic-gravitational interaction), and the conversion of gravitophotons into quintessence particles (gravitational-gravitational interaction).

quantized bang According to Heim, the universe did not evolve from a hot big bang, but instead, spacetime was discretized from the very beginning, and such no infinitely small or infinitely dense space existed. Instead, when the size of a single metron covered the whole (spherical volume) universe, this was considered the beginning of this physical universe. That condition can be considered as the mathematical initial condition and, when in- 
serted into Heim's equation for the evolution of the universe, does result in the initial diameter of the original universe [1]. Much later, when the metron size had decreased far enough, did matter come into existence as a purely geometrical phenomenon.

\section{sieve operator see $\rightarrow$ transformtion operator}

transformation operator or sieve operator (Sieboperator) The direct translation of Heim's terminology would be sieve-selector. A transformation operator, however, converts a photon into a gravitophoton by making the coordinate $\xi_{4}$ Euclidean.

\section{vacuum particle responsible for the accel-} eration of the universe, also termed quintessence particle The vacuum particle represents the sixth fundamental interaction and is a repulsive gravitational force whose gravitational coupling constant is given by $4.3565 \times 10^{-18} G$, see Appendix B.

\section{References}

1. Dröscher, W., J. Häuser: Future Space Propulsion Based on Heim's Field Theory, AIAA 2003-4990, AIAA/ASME/SAE/ASE, Joint Propulsion Conference \& Exhibit, Huntsville, AL, 21-24 July, 2003, 25 pp., see also www.uibk.ac.at/c/cb/cb26 and www.cle. de/hpcc.

2. Dröscher, W., J. Häuser: Physical Principles of Advanced Space Transportation based on Heim's Field Theory, AIAA/ASME/SAE/ASE, 38 th Joint Propulsion Conference \& Exhibit, Indianapolis, Indiana, 7-10 July, 2002, AIAA 2002-2094, 21 pp., see also www.cle.de/hpcc and www.uibk.ac.at/c/cb/cb26.

3. Millis, M.G.: (ed.), NASA Breakthrough Propulsion Physics, Workshop Proceedings, NASA/CP-1999 -208694 and NASA-TM-2004-213082, Prospects for Breakthrough Propulsion from Physics, May 2004, www.grc.nasa.gov/WWW/bpp/TM-2004213082.htm.

4. Heim, B.: Vorschlag eines Weges einer einheitlichen Beschreibung der Elementarteilchen, Zeitschrift für Naturforschung, 32a, 1977, pp. 233-243.

5. Penrose, R.: The Small, the Large and the Human Mind, Cambridge University Press, 1997.

6. Heim, B.: Elementarstrukturen der Materie, Band 1, Resch Verlag, 3rd ed., Innsbruck, 1998.

7. Heim, B.: Elementarstrukturen der Materie, Band 2, Resch Verlag, 2nd ed., Innsbruck, 1984.
8. Heim, B.: Ein Bild vom Hintergrund der Welt, in A. Resch (ed.) Welt der Weltbilder, Imago Mundi, Band 14, Resch Verlag, Innsbruck, 1994.

9. Heim, B. and W. Dröscher: Strukturen der physikalischen Welt und ihrer nichtmateriellen Seite, Innsbruck, Resch Verlag, 1996.

10. Heim, B., Flugkörper, Heft 6-8, (in German only) 1959.

11. Smolin, L., Atoms of Space and Time, Scientific American, January 2004.

12. Ludwiger, von I., Grünert, K., Zur Herleitung der Heim'schen Massenformel, IGW, Innsbruck University, 2003, 81 pp., paper (in German only) available in PDF format at:

http://www.uibk.ac.at/c/cb/cb26/heim/index.html.

13. Cline, D.B.: The Search for Dark Matter, Scientific American, February 2003.

14. Lawrie, I.D.: A Unified Grand Tour of Theoretical Physics, 2nd ed., IoP 2002.

15. Harris, E.G.: Modern Theoretical Physics, Vol I, Wiley\&Sons, 1975.

16. Landau, L., Lifschitz, E., Lehrbuch der Theoretischen Physik, Volume IV, §114, 1991.

17. Veltman, M., Facts and Mysteries in Elementary Particle Physics, World Scientific, 2003.

18. Penrose, R., The Emperor's New Mind, Chap. 5, Vintage, 1990.

19. Ashford, D., Spaceflight Revolution, Imperial College Press, 2002.

20. Wentzel, G., Quantum Theory of Fields, Interscience Publishers, 1949.

21. Jordan, P., Das Bild der mordernen Physik, Strom Verlag Hamburg-Bergedorf, 1947.

22. Woan, G., The Cambridge Handbook of Physics Formulas, Cambridge University Press, 2000.

23. Hawking, S., Penrose, R., The Nature of Space and Time, Princeton University Press, Chap. 4, 1996.

24. Ashtekar, A., et al., Background Independent Quantum Gravity:A Status Report, 125 pp., arXiv:grqc/0404018 v1, 5 April 2004.

25. Rovelli, C., Quantum Gravity, Chap.1, 329 pp., draft version 30 December 2003, to be published by Cambridge Univ. Press, 2004.

26. Rovelli, C., Loop Quantum Gravity, Physics World, November 2003.

27. Rovelli, C., Quantum Spacetime, Chap. 4 in Physics Meets Philosophy at the Planck Scale, eds. C. Callendar, N. Huggett, Cambridge Univ. Press, 2001.

28. Bojowald, M., Quantum Gravity and the Big Bang, arXiv:astro-ph/0309478, 2003.

29. Thiemann, T., Lectures on Loop Quantum Gravity, arXiv:gr-qc/0210094 v1, 28 Oct. 2002. 